(n)

trobertivier Journal of Nonlinear Mathematical Physics

\title{
Integrable systems from inelastic curve flows in 2- and 3- dimensional Minkowski space
}

Kivilcim Alkan, Stephen C. Anco

To cite this article: Kivilcim Alkan, Stephen C. Anco (2016) Integrable systems from inelastic curve flows in 2- and 3- dimensional Minkowski space, Journal of Nonlinear Mathematical Physics 23:2, 256-299, DOI:

https://doi.org/10.1080/14029251.2016.1175822

To link to this article: https://doi.org/10.1080/14029251.2016.1175822

Published online: 04 January 2021 


\title{
Integrable systems from inelastic curve flows in 2- and 3- dimensional Minkowski space
}

\author{
Kivilcim Alkan \\ Department of Mathematics \\ Izmir Institution of Technology \\ Izmir 35430 Turkey \\ kivilcimalkan@iyte.edu.tr \\ Stephen C. Anco \\ Department of Mathematics and Statistics \\ Brock University \\ St. Catharines, ON L2S3A1, Canada \\ sanco@brocku.ca
}

Received 6 December 2015

Accepted 24 February 2016

\begin{abstract}
Integrable systems are derived from inelastic flows of timelike, spacelike, and null curves in 2- and 3- dimensional Minkowski space. The derivation uses a Lorentzian version of a geometrical moving frame method which is known to yield the modified Korteveg-de Vries (mKdV) equation and the nonlinear Schrödinger (NLS) equation in 2- and 3- dimensional Euclidean space, respectively. In 2-dimensional Minkowski space, timelike/spacelike inelastic curve flows are shown to yield the defocusing $\mathrm{mKdV}$ equation and its bi-Hamiltonian integrability structure, while inelastic null curve flows are shown to give rise to Burgers' equation and its symmetry integrability structure. In 3-dimensional Minkowski space, the complex defocusing mKdV equation and the NLS equation along with their bi-Hamiltonian integrability structures are obtained from timelike inelastic curve flows, whereas spacelike inelastic curve flows yield an interesting variant of these two integrable equations in which complex numbers are replaced by hyperbolic (split-complex) numbers.
\end{abstract}

Keywords: curve flow; integrable system; Minkowski plane; Minkowski space

\section{Introduction}

There is an interesting geometric relationship between integrable systems and non-stretching (inelastic) geometrical flows of curves in various geometric spaces. For example, in the Euclidean plane, inelastic curve flows yield [12] the focusing mKdV equation $u_{t}=u_{x x x}+\frac{3}{2} u^{2} u_{x}$ where $x$ is the arclength of the curve and $u$ is the curvature invariant of the curve, with the geometrical motion of the curve being given by the vortex patch equation. In Euclidean space, inelastic curve flows whose geometrical motion is given by the vortex filament equation [15] and its axial generalization [11] respectively yield $[4,11,15,16,18]$ the focusing NLS equation $-i u_{t}=u_{x x}+\frac{1}{2}|u|^{2} u$ and the focusing complex mKdV equation $u_{t}=u_{x x x}+\frac{3}{2}|u|^{2} u_{x}$. Here $u=\kappa \exp \left(i \int \tau d x\right)$ is the Hasimoto variable [15] which is a covariant of the curve defined in terms of the curvature and torsion invariants $\kappa$ and $\tau$ up to arbitrary (constant) phase rotations $u \rightarrow e^{i \phi} u$.

The derivation of these integrable systems from the underlying curve flows is based on a moving frame method that uses a Frenet frame [13] in the case of curves in the Euclidean plane and a parallel frame [5] in the case of curves in Euclidean space. Parallel frames differ from a Frenet 
frame by a gauge transformation given by a rotation of the two normal vectors (in the normal plane of the curve) such that, at each point, their derivative along the curve is purely tangential. In both the case of the Euclidean plane and Euclidean space, the components of the Cartan matrix of the frame define the flow variable $u$, and the Cartan structure equations of the frame yield a Lax pair as well as a pair of Hamiltonian operators, which provide the integrability structure of the flows on $u$. In addition, in the case of space curves, the $U(1)$ phase-rotation invariance of the flows on $u$ geometrically corresponds to the fact that a parallel frame is determined by a curve only up to the action of arbitrary rigid $S O(2)$ rotations on the normal vectors in the frame, whereas a Frenet frame is determined uniquely by a curve.

In this paper, integrable systems are derived in an analogous way from inelastic curve flows in the Minkowski plane and in Minkowski space [21]. Several new results are obtained by considering timelike curves, spacelike curves, and null curves. The paper is organized as follows.

In Sec. 2, a Lorentzian version of a Frenet frame is applied to inelastic curve flows in the Minkowski plane. Geometrical inelastic flows of timelike and spacelike curves are found to yield the defocusing $\mathrm{mKdV}$ equation and its bi-Hamiltonian structure. In contrast, for null curves, a Frenet frame does not exist and instead a null frame is introduced. Geometrical inelastic flows of null curves are shown to yield Burger's equation and the Cole-Hopf transformation under which Burger's equation is mapped into the heat equation. This geometric realization of Burger's equation is new. (See Ref. [10] for related work.) Frame formulations of inelastic flows of timelike, spacelike, and null curves has appeared previously in Ref. [14].

In Sec. 3, starting from a Frenet frame, a Lorentzian counterpart of a parallel frame is introduced for timelike curves in 3-dimensional Minkowski space. The normal plane of a timelike curve is spacelike, and so a Lorentzian parallel frame is determined by a curve up to the action of rigid $S O(2)$ rotations, similarly to the case of Euclidean space. Consequently, the Cartan matrix of this frame yields the same complex-valued Hasimoto variable as in the case of Euclidean space. For timelike curves undergoing an inelastic geometrical flow given by a timelike version of the vortex filament equation and its axial generalization, the Cartan structure equations of the Lorentzian parallel frame are shown to yield the defocusing NLS equation and the defocusing complex mKdV equation along with their bi-Hamiltonian integrability structure. A similar derivation of the defocusing NLS equation has appeared in Ref. [8] without, however, deriving the bi-Hamiltonian structure of this equation or considering the defocusing complex $\mathrm{mKdV}$ equation. Parallel frames for non-null curves have been considered previously in Ref. [22].

In Sec. 4, spacelike curves in Minkowski space are considered. Because the normal plane of a spacelike curve is timelike, two different cases arise depending on whether the principal normal vector of the curve is non-null or null.

When the principal normal vector of a spacelike curve is non-null, a Frenet frame exists which is used to define a Lorentzian parallel frame by a gauge transformation given by a boost (hyperbolic rotation) of the two normal vectors. Consequently, the resulting frame is determined by the curve only up to the action of rigid $S O(1,1)$ boosts. These boosts comprise a group of hyperbolic rotations, and the Hasimoto variable arising from the Cartan matrix of this frame is no longer a complex variable but instead is a hyperbolic generalization [7] based on the split-complex numbers defined by $j^{2}=1$ and $\bar{j}=-j$. For spacelike curves undergoing an inelastic geometrical flow given by a spacelike generalization of the vortex filament equation and its axial generalization, the Cartan structure equations of a non-null parallel frame are shown to yield variants of the defocusing NLS equation and the defocusing complex mKdV equation, along with their bi-Hamiltonian integrability 
structure, in which $i$ is replaced by $j$. Such integrable systems have been derived previously in the literature [6] by purely algebraic methods. Our results provide an interesting geometric realization for these systems. A special case of the $S O(1,1)$-invariant complex $\mathrm{mKdV}$ equation has been derived in Ref. [9] using similar geometrical methods, and a system equivalent to the $S O(1,1)$-invariant NLS equation appears in Ref. [8] in a less geometric form, without any results on the bi-Hamiltonian structure of these equations.

When a spacelike curve has a null principal normal vector, a complete Frenet frame does not exist. Instead a null frame is introduced for the normal plane. Then a Lorentzian parallel frame is defined by a gauge transformation such that the derivative of the pair of null normal vectors in the frame is purely tangential to the curve. The gauge transformation acts as a scaling on these null normal vectors, and consequently the resulting frame is determined by the curve only up to the action of rigid scalings (where the two null vectors scale reciprocally to each other). Because the principal normal vector of the curve is constrained to be null, the Cartan matrix has one fewer component than in the case when the principal normal vector is non-null. Interestingly, the Cartan structure equations are found to yield Burgers' equation and the KdV equation, as well as the heat equation and the Airy equation. These results are new. The corresponding geometrical inelastic curve flows are shown to be given by variants of a heat map equation and an Airy map equation.

Related work on null curve flows in 3- and 4- dimensional Minkowski space appears in Ref. $[14,19,20]$.

Some concluding remarks are made in Sec. 5.

\section{Curve flows and integrable systems in $\mathbb{R}^{1,1}$}

The Minkowski plane is a 2-dimensional vector space $\mathbb{R}^{1,1}$ equipped with a Lorentz-signature metric $\eta$ and a compatible volume form $\varepsilon$. A vector $\vec{v} \in \mathbb{R}^{1,1}$ is respectively timelike, spacelike, or null if its Minkowski norm $\eta(\vec{v}, \vec{v})$ is negative, positive, or zero. The set of all null vectors spans a pair of 1-dimensional null lines through the origin in $\mathbb{R}^{1,1}$, called the lightcone.

Up to a sign, the volume form is determined from the metric by the relation

$$
\varepsilon(\vec{v}, \vec{w})^{2}=\eta(\vec{v}, \vec{w})^{2}-\eta(\vec{v}, \vec{v}) \eta(\vec{w}, \vec{w})
$$

holding for any vectors $\vec{v}, \vec{w} \in \mathbb{R}^{1,1}$. Associated to the metric and the volume form is the Hodge dual operator $*$ defined by

$$
\eta(* \vec{v}, \vec{w})=\varepsilon(\vec{v}, \vec{w})
$$

which has the properties

$$
*(* \vec{v})=\vec{v}, \quad \eta(* \vec{v}, \vec{v})=0
$$

and

$$
\eta(* \vec{v}, \vec{w})=-\eta(* \vec{w}, \vec{v}), \quad \eta(* \vec{v}, * \vec{w})=-\eta(\vec{v}, \vec{w})
$$

holding for any vectors $\vec{v}, \vec{w} \in \mathbb{R}^{1,1}$. 
In inertial coordinates $X^{i}=\left(X^{0}, X^{1}\right)$, the Minkowski metric has the components $\eta_{i j}=\left(\begin{array}{cc}-1 & 0 \\ 0 & 1\end{array}\right)$ and the volume tensor has the components $\varepsilon_{i j}=\left(\begin{array}{cc}0 & 1 \\ -1 & 0\end{array}\right)$, where $X^{0}$ is a timelike coordinate and $X^{1}$ is a spacelike coordinate. The Hodge dual operator $*$ is given by the associated tensor $\varepsilon_{i}{ }^{j}=\varepsilon_{i k} \eta^{j k}$.

The isometries of the Minkowski plane consist of time and space translations and boosts. These transformations comprise the 3-dimensional Poincare group $I S O(1,1) \simeq S O(1,1) \ltimes \mathbb{R}^{2}$ (also called the inhomogeneous Lorentz group).

\subsection{Timelike/spacelike curves and Frenet frames}

Let $\vec{\gamma}(x)=\left(X^{0}(x), X^{1}(x)\right)$ be a curve in $\mathbb{R}^{1,1}$ such that

$$
\eta\left(\vec{\gamma}_{x}, \vec{\gamma}_{x}\right) \neq 0
$$

at every point $x$ on the curve. Such a curve is timelike if $\eta\left(\vec{\gamma}_{x}, \vec{\gamma}_{x}\right)<0$ or spacelike if $\eta\left(\vec{\gamma}_{x}, \vec{\gamma}_{x}\right)>0$. In both cases we hereafter choose $x$ to be the proper-time or proper-distance arclength parameter defined by

$$
\sqrt{\left|\eta\left(\vec{\gamma}_{x}, \vec{\gamma}_{x}\right)\right|}=1
$$

From relation (2.3), we note that the vectors $\vec{\gamma}_{x}$ and $* \vec{\gamma}_{x}$ are orthogonal in the Minkowski metric. Since $\vec{\gamma}_{x}$ is assumed to be non-null, then this implies $* \vec{\gamma}_{x}$ is also non-null. Hence, for a timelike/spacelike curve $\vec{\gamma}(x)$ in $\mathbb{R}^{1,1}$, this pair of vectors can be used to define a Lorentzian version of a Frenet frame [14]

$$
\begin{aligned}
& e_{\|}=\vec{\gamma}_{x}=T, \quad \text { unit timelike/spacelike tangent vector } \\
& e_{\perp}=* \vec{\gamma}_{x}=* T=N, \quad \text { unit spacelike/timelike normal vector }
\end{aligned}
$$

satisfying

$$
\begin{gathered}
\eta\left(e_{\|}, e_{\|}\right)=\mp 1, \quad \eta\left(e_{\perp}, e_{\perp}\right)= \pm 1, \\
\eta\left(e_{\|}, e_{\perp}\right)=0 .
\end{gathered}
$$

The Frenet equations for this frame (2.7) are easily derived as follows. First, from the $x$ derivative of equation (2.8), we have $\eta\left(e_{\| x}, e_{\|}\right)=0$ and $\eta\left(e_{\perp x}, e_{\perp}\right)=0$, which implies $e_{\| x}=u_{1} e_{\perp}$ and $e_{\perp x}=u_{2} e_{\|}$for some functions $u_{1}(x)$ and $u_{2}(x)$. Next, from the $x$-derivative of equation (2.9), we have $\eta\left(e_{\| x}, e_{\perp}\right)+\eta\left(e_{\perp x}, e_{\|}\right)=0$. Now substituting the previous expressions for the $x$-derivatives of $e_{\|}$and $e_{\perp}$, and then using the norm relations (2.8), we find $0=u_{1} \eta\left(e_{\perp}, e_{\perp}\right)+u_{2} \eta\left(e_{\|}, e_{\|}\right)=$ $\pm\left(u_{1}-u_{2}\right)$. This yields $u_{1}=u_{2}=u$ in both cases. Thus the Frenet equations are given by

$$
\left(\begin{array}{l}
e_{\|} \\
e_{\perp}
\end{array}\right)_{x}=\left(\begin{array}{ll}
0 & u \\
u & 0
\end{array}\right)\left(\begin{array}{l}
e_{\|} \\
e_{\perp}
\end{array}\right)
$$

where $u$ geometrically represents the Lorentzian curvature invariant of the curve $\vec{\gamma}(x)$, and where the Cartan matrix $\left(\begin{array}{ll}0 & u \\ u & 0\end{array}\right)$ belongs to the Lie algebra $\mathfrak{s o}(1,1)$ of the $S O(1,1)$ group of boost isometries in $\mathbb{R}^{1,1}$. 
We now consider curve flows $\vec{\gamma}(t, x)$ that locally preserve both the timelike/spacelike signature (2.5) of the curve and the proper time/distance normalization (2.6) of the arclength parameter. Such flows are called inelastic and are specified by a flow vector

$$
\vec{\gamma}_{t}=h_{\|} e_{\|}+h_{\perp} e_{\perp}
$$

expressed in terms of tangential and normal components in the Frenet frame (2.7). The Frenet frame itself will be carried by the flow, according to the equations

$$
\left(\begin{array}{l}
e_{\|} \\
e_{\perp}
\end{array}\right)_{t}=\left(\begin{array}{ll}
0 & \omega \\
\omega & 0
\end{array}\right)\left(\begin{array}{l}
e_{\|} \\
e_{\perp}
\end{array}\right)
$$

which arise from equations (2.8)-(2.9) similarly to the derivation of the Frenet equations (2.10). Here $\omega$ geometrically represents a Lorentzian invariant of the curve flow, and the Cartan matrix $\left(\begin{array}{cc}0 & \omega \\ \omega & 0\end{array}\right)$ belongs to the Lie algebra $\mathfrak{s o}(1,1)$.

The Frenet equations (2.10) and the flow equations (2.12) are related by the compatibility condition that the mixed $t, x$-derivatives of the Frenet frame (2.7) are equal. Since the Cartan matrices commute, this yields

$$
u_{t}=\omega_{x}
$$

Likewise, the tangent vector (2.7a) and the flow vector (2.11) of the curve are related by the compatibility condition that the mixed $t, x$-derivatives of $\vec{\gamma}(t, x)$ are equal, which gives

$$
h_{\perp x} e_{\perp}+h_{\perp} e_{\perp x}+h_{\| x} e_{\|}+h_{\|} e_{\| x}=e_{\| t} .
$$

After substituting the Frenet equations (2.10) and the flow equations (2.12) for the derivatives of $e_{\|}$ and $e_{\perp}$, we obtain

$$
e_{\|}\left(h_{\| x}+u h_{\perp}\right)+e_{\perp}\left(h_{\perp x}+u h_{\|}-\omega\right)=0 .
$$

Hence, from the vanishing of the coefficients of the frame vectors $e_{\|}$and $e_{\perp}$, we find

$$
\begin{gathered}
\omega=h_{\perp x}+u h_{\|}, \\
h_{\| x}=-u h_{\perp} .
\end{gathered}
$$

These compatibility equations (2.16)-(2.17) and (2.13) are the Cartan structure equations of the Frenet frame. They have a natural operator formulation as follows. We use equation (2.17) to express $h_{\|}=-D_{x}^{-1}\left(u h_{\perp}\right)$, so then (2.16) becomes

$$
\omega=h_{\perp x}-u D_{x}^{-1}\left(u h_{\perp}\right) .
$$

Hence, the Cartan structure equations reduce to the system (2.18) and (2.13).

Recall that an operator $\mathscr{H}$ is Hamiltonian iff it defines an associated Poisson bracket

$$
\{\mathfrak{H}, \mathfrak{G}\}_{\mathscr{H}}=\int(\delta \mathfrak{H} / \delta u) \mathscr{D}(\delta \mathfrak{G} / \delta u) d x
$$

obeying skew-symmetry $\{\mathfrak{H}, \mathfrak{G}\}_{\mathscr{H}}+\{\mathfrak{G}, \mathfrak{H}\}_{\mathscr{H}}=0$ and the Jacobi identity $\left\{\{\mathfrak{H}, \mathfrak{G}\}_{\mathscr{H}}, \mathfrak{F}\right\}_{\mathscr{H}}+$ cyclic $=0$ for all functionals $\mathfrak{H}, \mathfrak{G}, \mathfrak{F}$ on the $x$-jet space $J^{\infty}$ of the variable $u$, where $\delta / \delta u$ denotes 
the standard Euler operator. The formal inverse of a Hamiltonian operator defines a symplectic operator. Compatibility of a Hamiltonian operator $\mathscr{H}$ and a symplectic operator $\mathscr{J}$ is the statement that every linear combination $c_{1} \mathscr{H}+c_{2} \mathscr{J}^{-1}$ is a Hamiltonian operator, or equivalently that $c_{1} \mathscr{H}^{-1}+c_{2} \mathscr{J}$ is a symplectic operator.

Theorem 1. For timelike or spacelike inelastic curve flows in $\mathbb{R}^{1,1}$, the curve invariant $u$ satisfies the system

$$
\begin{gathered}
u_{t}=\mathscr{H}(\omega), \quad \mathscr{H}=D_{x} \\
\omega=\mathscr{J}\left(h_{\perp}\right), \quad \mathscr{J}=D_{x}-u D_{x}^{-1} u
\end{gathered}
$$

where $\mathscr{H}$ and $\mathscr{J}$ are a pair of compatible Hamiltonian and symplectic operators. Composition of these operators yields the evolution equation

$$
u_{t}=\mathscr{R}\left(h_{\perp}\right)
$$

for $u$ in terms of the normal component $h_{\perp}$ of the flow, where

$$
\mathscr{R}=\mathscr{H} \mathscr{J}=D_{x}^{2}-u^{2}-u_{x} D_{x}^{-1} u
$$

is a hereditary recursion operator.

This theorem is a counterpart of a similar result (see e.g. [3,23]) known for inelastic curve flows in the Euclidean plane and can be proved by the same methods. In particular, compared to the Euclidean case, the Hamiltonian operator (2.20) is exactly the same while the symplectic operator (2.21) differs only by the sign of the nonlocal term.

As a consequence of the compatibility of this pair of operators (2.20) and (2.21), their obvious invariance under $x$-translations can be used to derive a hierarchy of flows starting from a root flow $u_{t}=u_{x}$ corresponding to the generator of $x$-translations on $u$, due to a general theorem of Magri [17]. This leads to the following main result.

Theorem 2. There is a hierarchy of integrable bi-Hamiltonian flows on $u(t, x)$ given by

$$
\begin{aligned}
u_{t}=\mathscr{R}^{n}\left(u_{x}\right) & =\mathscr{H}\left(\boldsymbol{\delta} \mathfrak{H}^{(n)} / \delta u\right), \quad n=0,1,2, \ldots \\
& =\mathscr{E}\left(\delta \mathfrak{H}^{(n-1)} / \delta u\right), \quad n=1,2, \ldots
\end{aligned}
$$

(called the $+n$ flow) in terms of Hamiltonians $\mathfrak{H}^{(n)}=\int H^{(n)} d x$ where

$$
\mathscr{H}=D_{x}, \quad \mathscr{E}=\mathscr{R} \mathscr{H}=D_{x}{ }^{3}-u^{2} D_{x}-u_{x} D_{x}^{-1} u D_{x}
$$

are compatible Hamiltonian operators, and where

$$
H^{(n)}=(1+2 n)^{-1} D_{x}^{-1}\left(u \mathscr{R}^{n}\left(u_{x}\right)\right), \quad n=0,1,2, \ldots
$$

are local Hamiltonian densities.

The expression for the Hamiltonians (2.26) in this theorem arises from a scaling formula derived in Ref. [1]. 
The +1 flow in the hierarchy (2.24) is explicitly given by

$$
u_{t}=u_{x x x}-\frac{3}{2} u^{2} u_{x}
$$

which is the defocusing $\mathrm{mKdV}$ equation. It has the explicit bi-Hamiltonian structure

$$
u_{t}=\mathscr{H}\left(\delta \mathfrak{H}^{(1)} / \delta u\right)=\mathscr{E}\left(\delta \mathfrak{H}^{(0)} / \delta u\right)
$$

in terms of the Hamiltonian densities

$$
H^{(0)}=\frac{1}{2} u^{2}, \quad H^{(1)}=-\frac{1}{2}\left(u_{x}^{2}+\frac{1}{4} u^{4}\right)
$$

(modulo trivial densities given by total $x$-derivatives).

The entire hierarchy (2.24) of flows corresponds to a hierarchy of commuting vector fields

$$
\mathrm{X}^{(n)}=\mathscr{R}^{n}\left(u_{x}\right) \partial_{u}, \quad n=0,1,2, \ldots
$$

with the root vector field being the generator of $x$-translations, $\mathrm{X}^{(0)}=u_{x} \partial_{u}$. Recall that a vector field is Hamiltonian iff its prolongation on the $x$-jet space $J^{\infty}$ satisfies $\operatorname{prX\mathfrak {G}}=\{\mathfrak{G}, \mathfrak{H}\} \mathscr{H}$ for some functional $\mathfrak{H}$, where $\mathfrak{G}$ is an arbitrary functional. Theorem 2 shows that all of the vector fields in this hierarchy are Hamiltonian, as given by $\operatorname{prX}{ }^{(n)} \mathfrak{G}=\left\{\mathfrak{G}, \mathfrak{H}^{(n)}\right\} \mathscr{H}$, where $\mathfrak{H}^{(n)}=\int H^{(n)} d x$ is the functional with the Hamiltonian density (2.26). Additionally, each vector field except for $\mathrm{X}^{(0)}=$ $u_{x} \partial_{u}$ is bi-Hamiltonian, due to $\operatorname{prX}^{(n)} \mathfrak{G}=\left\{\mathfrak{G}, \mathfrak{H}^{(n-1)}\right\}_{\mathscr{E}}$ for all $n \geq 1$. Since the hierarchy for all $n \geq 0$ is commuting, every Hamiltonian vector field (2.30) is the generator of a symmetry for the defocusing $\mathrm{mKdV}$ equation (2.27), and every associated Hamiltonian (2.26) is a conserved density for the defocusing $\mathrm{mKdV}$ equation (2.27).

Proposition 1. Each flow in the hierarchy (2.24) determines an inelastic timelike/spacelike curve flow

$$
\vec{\gamma}_{t}=h_{\|}^{(n)} T+h_{\perp}^{(n)} N, \quad n=1,2, \ldots
$$

whose tangential and normal components are given by

$$
h_{\|}^{(n)}=(2 n-1) H^{(n-1)}, \quad h_{\perp}^{(n)}=\mathscr{R}^{n-1}\left(u_{x}\right), \quad n=1,2, \ldots
$$

where

$$
T=\vec{\gamma}_{x}, \quad N=* \vec{\gamma}_{x}
$$

The components (2.32) of these flows are functions of the curve invariant $u$ and its $x$-derivatives, so thus the equation of motion (2.31) are invariant under the isometry group ISO $(1,1)$ of the Minkowski plane.

Each equation of motion (2.31) describes a geometric non-stretching motion of the curve. The curve flow $n=1$ corresponding to the defocusing $\mathrm{mKdV}$ equation (2.27) is given by

$$
\vec{\gamma}_{t}=-\frac{1}{2} u^{2} T+u_{x} N .
$$

This equation of motion is a Lorentzian version of the vortex patch equation. It can be expressed entirely in terms of $\vec{\gamma}_{x}, * \vec{\gamma}_{x}$, and their $x$-derivatives through the Frenet equations (2.10) as follows. 
We have

$$
u= \pm \eta\left(e_{\| x}, e_{\perp}\right)= \pm \eta\left(* \vec{\gamma}_{x}, \vec{\gamma}_{x x}\right), \quad u^{2}= \pm \eta\left(e_{\| x}, e_{\| x}\right)= \pm \eta\left(\vec{\gamma}_{x x}, \vec{\gamma}_{x x}\right)
$$

and hence

$$
u_{x}= \pm \eta\left(* \vec{\gamma}_{x}, \vec{\gamma}_{x x x}\right)
$$

using the relation (2.3). Thus the equation of motion (2.34) becomes

$$
\vec{\gamma}_{t}=-\frac{1}{2} \eta\left(* \vec{\gamma}_{x}, \vec{\gamma}_{x x}\right)^{2} \vec{\gamma}_{x} \pm \eta\left(* \vec{\gamma}_{x}, \vec{\gamma}_{x x x}\right) * \vec{\gamma}_{x}, \quad \eta\left(\vec{\gamma}_{x}, \vec{\gamma}_{x}\right)=\mp 1
$$

We can simplify this equation of motion further by using the frame expansion

$$
\vec{v}= \pm\left(\eta\left(\vec{v}, e_{\perp}\right) e_{\perp}-\eta\left(\vec{v}, e_{\|}\right) e_{\|}\right)
$$

holding for any vector $\vec{v} \in \mathbb{R}^{1,1}$. This yields

$$
\vec{\gamma}_{t}=\mp \frac{3}{2} \eta\left(\vec{\gamma}_{x x}, \vec{\gamma}_{x x}\right) \vec{\gamma}_{x}+\vec{\gamma}_{x x x}, \quad \eta\left(\vec{\gamma}_{x}, \vec{\gamma}_{x}\right)=\mp 1
$$

which is a Lorentzian version of the non-stretching mKdV map equation [2], in the Minkowski plane.

\subsection{Null curves and null frames}

Let $\vec{\gamma}(x)=\left(X^{0}(x), X^{1}(x)\right)$ be a null curve in $\mathbb{R}^{1,1}$, satisfying

$$
\eta\left(\vec{\gamma}_{x}, \vec{\gamma}_{x}\right)=0
$$

at every point $x$ on the curve. Here, in contrast to the case of non-null curves, the parameter $x$ is arbitrary and cannot be normalized solely in terms of $\vec{\gamma}_{x}$.

Moreover, the vectors $\vec{\gamma}_{x}$ and $* \vec{\gamma}_{x}$ are now parallel, since we have $0=\eta\left(\vec{\gamma}_{x}, \vec{\gamma}_{x}\right)=-\eta\left(* \vec{\gamma}_{x}, * \vec{\gamma}_{x}\right)=$ $\varepsilon\left(* \vec{\gamma}_{x}, \vec{\gamma}_{x}\right)$, from the relations (2.2) and (2.4), where antisymmetry of $\varepsilon$ directly implies $* \vec{\gamma}_{x}=a \vec{\gamma}_{x}$ for some function $a(x)$. Similarly, the vectors $\vec{\gamma}_{x}$ and $\vec{\gamma}_{x x}$ are parallel, since the $x$-derivative of $\eta\left(\vec{\gamma}_{x}, \vec{\gamma}_{x}\right)=$ 0 yields $\eta\left(\vec{\gamma}_{x}, \vec{\gamma}_{x x}\right)=0$ from which we obtain $\varepsilon\left(* \vec{\gamma}_{x}, \vec{\gamma}_{x x}\right)=-\eta\left(\vec{\gamma}_{x}, \vec{\gamma}_{x x}\right)=0$ through the relations (2.2) and (2.4). Antisymmetry of $\varepsilon$ then implies $\vec{\gamma}_{x x}=b * \vec{\gamma}_{x}$ for some function $b(x)$, and thus $\vec{\gamma}_{x x}$ is parallel to $* \vec{\gamma}_{x}$ which itself is parallel to $\vec{\gamma}_{x}$.

Hence, for defining a frame, we need another vector, linearly independent of $\vec{\gamma}_{x}$. One natural choice is to use a null vector on the opposite side of the lightcone. If $\vec{v}$ is a null vector in $\mathbb{R}^{1,1}$, let $\mathscr{N}$ be a linear map that produces a null vector $\mathscr{N}(\vec{v})$ such that $\eta(\mathscr{N}(\vec{v}), \vec{v})=-1$. Note this implies $\vec{v}+\mathscr{N}(\vec{v})$ is a timelike vector, since $\eta(\vec{v}+\mathscr{N}(\vec{v}), \vec{v}+\mathscr{N}(\vec{v}))=2 \eta(\vec{v}, \mathscr{N}(\vec{v}))=-2$. Hence the null vectors $\vec{v}$ and $\mathscr{N}(\vec{v})$ are spatial reflections of each other with respect to this timelike vector in $\mathbb{R}^{1,1}$, with the reflection being given by $\vec{v}-\frac{1}{2}(\vec{v}+\mathscr{N}(\vec{v}))=\frac{1}{2}(\vec{v}-\mathscr{N}(\vec{v}))=-\left(\mathscr{N}(\vec{v})-\frac{1}{2}(\vec{v}+\mathscr{N}(\vec{v}))\right)$ where $\vec{v}-\mathscr{N}(\vec{v})$ is a spacelike vector orthogonal to $\vec{v}+\mathscr{N}(\vec{v})$. Also note that a change in the normalization $\eta(\mathscr{N}(\vec{v}), \vec{v})=-1$ only produces a scaling of the null vector $\mathscr{N}(\vec{v})$. 
A null frame for a null curve $\vec{\gamma}(x)$ in $\mathbb{R}^{1,1}$ can then be defined by

$$
\begin{aligned}
& e_{+}=\vec{\gamma}_{x}=T, \quad \text { null tangent vector } \\
& e_{-}=\mathscr{N}\left(\vec{\gamma}_{x}\right)=\mathscr{N}(T), \quad \text { null opposite vector }
\end{aligned}
$$

with

$$
\begin{gathered}
\eta\left(e_{+}, e_{+}\right)=\eta\left(e_{-}, e_{-}\right)=0 \\
\eta\left(e_{+}, e_{-}\right)=-1
\end{gathered}
$$

where these properties (2.42)-(2.43) uniquely determine $e_{-}$when $e_{+}$is given.

The Frenet equations for this frame (2.41) are easily derived by the same steps used in the case of a Frenet frame. First, the $x$-derivative of equation (2.42) yields $\eta\left(e_{+x}, e_{+}\right)=0$ and $\eta\left(e_{-x}, e_{-}\right)=$ 0 . This implies $e_{ \pm x}=u_{ \pm} e_{ \pm}$for some functions $u_{ \pm}(x)$. Next, the $x$-derivative of equation (2.43) combined with the previous expressions for the $x$-derivatives of $e_{ \pm}$gives $0=\left(u_{+}+u_{-}\right) \eta\left(e_{+}, e_{-}\right)=$ $-\left(u_{+}+u_{-}\right)$, and hence we have $u_{+}=-u_{-}=u$. The Frenet equations are thus given by

$$
\left(\begin{array}{l}
e_{+} \\
e_{-}
\end{array}\right)_{x}=\left(\begin{array}{cc}
u & 0 \\
0 & -u
\end{array}\right)\left(\begin{array}{l}
e_{+} \\
e_{-}
\end{array}\right)
$$

where the Cartan matrix $\left(\begin{array}{cc}u & 0 \\ 0 & -u\end{array}\right)$ belongs to the Lie algebra of the abelian group of null boosts in $\mathbb{R}^{1,1}$. These equations (2.44) are preserved if the normalization (2.43) of the null frame is changed. However, if the null parameter $x$ is changed by reparameterizing the curve, then the equations (2.44) undergo a gauge transformation. In particular, for $x \rightarrow \tilde{x}(x)$, with $\tilde{x}^{\prime} \neq 0$, we have $u \rightarrow \tilde{u}=\left(1 / \tilde{x}^{\prime}\right) u+$ $\left(1 / \tilde{x}^{\prime}\right)^{\prime}$. By restricting such reparameterizations to affine transformations $\tilde{x}=\alpha x+\beta$, where $\alpha \neq$ 0 and $\beta$ are constants, we see that $u$ only undergoes a scaling by $\alpha$. Therefore, $u$ geometrically represents a relative affine invariant of the parameterized null curve.

We now consider inelastic null curve flows $\vec{\gamma}(t, x)$, in which both the null signature $\eta\left(\vec{\gamma}_{x}, \vec{\gamma}_{x}\right)=0$ and the null parameter $x$ of the curve are locally preserved by the flow. Such flows are specified by a flow vector

$$
\vec{\gamma}_{t}=h_{+} e_{+}+h_{-} e_{-}
$$

expressed in terms of the null frame (2.41). The null frame will be carried by the flow, such that

$$
\left(\begin{array}{l}
e_{+} \\
e_{-}
\end{array}\right)_{t}=\left(\begin{array}{cc}
\omega & 0 \\
0 & -\omega
\end{array}\right)\left(\begin{array}{l}
e_{+} \\
e_{-}
\end{array}\right)
$$

where the Cartan matrix belongs to the Lie algebra of the abelian group of null boosts in $\mathbb{R}^{1,1}$. These equations (2.46) arise from the null frame equations (2.42)-(2.43) similarly to the derivation of the Frenet equations (2.44) and are therefore preserved if the normalization (4.90) of the null frame is changed. Consequently, $\omega$ represents a relative affine invariant of the time-parameterized curve flow.

The Frenet equations (2.44) and the null flow equations (2.46) are related by the compatibility condition that the mixed $t, x$-derivatives of the null frame (2.41) are equal. Likewise, the tangent vector (2.41a) and the flow vector (2.45) of the curve are related by the compatibility condition that 
the mixed $t, x$-derivatives of $\vec{\gamma}(t, x)$ are equal. By the same steps used in the spacelike/timelike case, these conditions yield

$$
\begin{gathered}
u_{t}=\omega_{x} \\
\omega=h_{+x}+u h_{+} \\
h_{-x}=u h_{-}
\end{gathered}
$$

which are the Cartan structure equations of the null frame.

From equation (2.49), we naturally obtain a potential variable related to the variable $u$ by

$$
v_{x}=h_{-x} / h_{-}=u, \quad v=\ln h_{-}=\int u d x
$$

up to the gauge freedom $v \rightarrow v+f(t)$. The remaining equations (2.47) and (2.48) then become

$$
v_{t}=\omega=h_{+x}+v_{x} h_{+}
$$

after a suitable gauge transformation is used to absorb an arbitrary integration constant (which is a function of $t$ ). Note, since $u$ has the geometrical meaning of a relative affine invariant of the curve under reparameterizations $x \rightarrow \alpha x+\beta$, where $\alpha \neq 0$ and $\beta$ are constants, we see that $v=\int u d x$ is invariant up to an additive constant. Therefore $v$ represents an affine covariant of the curve.

The system (2.50)-(2.51) has an interesting operator structure, involving a recursion operator, as follows.

Theorem 3. For inelastic null curve flows in $\mathbb{R}^{1,1}$, the affine covariant $v=\int u d x$ satisfies the equation

$$
v_{t}=\mathscr{R}\left(h_{+}\right), \quad \mathscr{R}=D_{x}+v_{x}
$$

in terms of the tangential component $h_{+}$of the flow, where $\mathscr{R}$ is the hereditary recursion operator for Burgers' equation in potential form.

This recursion operator $\mathscr{R}$ can be used to derive a hierarchy of flows starting from a root flow $v_{t}=v_{x}$ corresponding to the generator of $x$-translations on $v$. The following main result shows that the odd flows have a gradient-energy structure, while the even flows have a Hamiltonian structure.

Theorem 4. There is a hierarchy of integrable flows on $v(t, x)$ given by

$$
\begin{aligned}
v_{t} & =\mathscr{R}^{n}\left(v_{x}\right), \quad n=1,2, \ldots \\
& = \begin{cases}-\exp (-2 v) \delta \mathfrak{H}^{(l)} / \delta v, \quad l=(n+1) / 2, & n=1,3, \ldots \\
\mathscr{H}\left(\delta \mathfrak{H}^{(l)} / \delta v\right), \quad l=n / 2, & n=2,4, \ldots\end{cases}
\end{aligned}
$$

(called the $+n$ flow) in terms of energies/Hamiltonians $\mathfrak{H}^{(n)}=\int H^{(n)} d x$, where

$$
\mathscr{H}=-\exp (-v) D_{x} \exp (-v)
$$

is a Hamiltonian operator, and where

$$
H^{(l)}=\frac{1}{2}(-1)^{l-1}\left(D_{x}^{l} \exp (v)\right)^{2}, \quad l=1,2, \ldots
$$

are local densities. 
The +1 flow in this hierarchy (2.53) is explicitly given by

$$
v_{t}=v_{x x}+v_{x}^{2}
$$

which is Burgers' equation in potential form. It has the gradient structure

$$
v_{t}=-\exp (-2 v) \delta \mathfrak{H}^{(1)} / \delta v
$$

in terms of the energy density

$$
H^{(1)}=\frac{1}{2} \exp (2 v) v_{x}^{2}
$$

We remark that this structure implies

$$
\frac{d \mathfrak{H}^{(1)}}{d t}=\int v_{t}\left(\delta H^{(1)} / \delta v\right) d x=-\int \exp (-2 v)\left(\delta H^{(1)} / \delta v\right)^{2} d x<0
$$

(modulo boundary terms in the integral) whereby the energy integral $\mathfrak{H}^{(1)}=\int \frac{1}{2} \exp (2 v) v_{x}^{2} d x>0$ is a positive, decreasing function of $t$. (This property can be used to show that solutions $v(t, x)$ of equation (2.57) having finite energy are dispersive.)

The odd part of the hierarchy (2.53) has a gradient-energy structure similar to this +1 flow. In contrast, the even part of the hierarchy is quite different.

The +2 flow is given by

$$
v_{t}=v_{x x x}+3 v_{x x} v_{x}+v_{x}^{3}
$$

which has the Hamiltonian structure

$$
v_{t}=\mathscr{H}\left(\delta \mathfrak{H}^{(1)} / \delta v\right)
$$

where the Hamiltonian is the same expression $\mathfrak{H}^{(1)}=\int \frac{1}{2} \exp (2 v) v_{x}^{2} d x$ as the energy integral appearing in the +1 flow. There is a similar Hamiltonian structure for the other even flows.

The entire hierarchy (2.53) of flows on $v(t, x)$ corresponds to a hierarchy of commuting vector fields

$$
\mathrm{X}^{(n)}=\mathscr{R}^{n}\left(v_{x}\right) \partial_{v}, \quad n=0,1,2, \ldots
$$

with the root vector field being the generator of $x$-translations, $\mathrm{X}^{(0)}=v_{x} \partial_{v}$. From Theorem 4, we see that all of the odd vector fields (i.e. $n=1,3, \ldots$ ) have a gradient structure, while all of the even vector fields (i.e. $n=2,4, \ldots$ ) have a Hamiltonian structure. Since the hierarchy is commuting, every vector field (2.63) in this hierarchy is the generator of a symmetry for Burgers' equation in potential form (2.57).

It is interesting to formulate the preceding results by using the variables $u$ and $h_{-}$which are related to $v$ by equation (2.50). This equation has exactly the form of the Hopf-Cole transformation, under which Burgers' equation can be mapped to the heat equation. 
In terms of $u$ and $h_{-}$, the vector fields (2.63) become

$$
\mathrm{X}^{(n)}=h_{+}^{(n)} \partial_{v}=g^{(n)} \partial_{u}=k^{(n)} \partial_{h_{-}}, \quad n=0,1,2, \ldots
$$

where

$$
h_{+}^{(n)}=\mathscr{R}^{n}\left(v_{x}\right), \quad g^{(n)}=D_{x} h_{+}^{(n)}=\mathscr{Q}^{n}\left(u_{x}\right), \quad k^{(n)}=h_{-} h_{+}^{(n)}=\mathscr{K}^{n}\left(h_{-x}\right)
$$

are given by the recursion operators

$$
\mathscr{R}=D_{x}+v_{x}, \quad \mathscr{Q}=D_{x} \mathscr{R} D_{x}^{-1}=D_{x}+u+u_{x} D_{x}^{-1}, \quad \mathscr{K}=\exp (v) \mathscr{R} \exp (-v)=D_{x}
$$

using equation (2.50). The odd and even vector fields in this hierarchy (2.64) will respectively inherit a gradient structure and a Hamiltonian structure through the variational relation

$$
\delta \mathfrak{H} / \delta v=-D_{x} \delta \mathfrak{H} / \delta u=\exp (v) \delta \mathfrak{H} / \delta h_{-} .
$$

This leads to the following result.

Theorem 5. The hierarchy of integrable flows (2.53) on $v(t, x)$ yields the equivalent hierarchy of linear flows on $h_{-}(t, x)$,

$$
\begin{aligned}
h_{-t} & =D_{x}{ }^{n}\left(h_{-x}\right), \quad n=1,2, \ldots \\
& = \begin{cases}-\delta \mathfrak{H}^{(l)} / \delta h_{-}, \quad l=(n+1) / 2, & n=1,3, \ldots \\
-D_{x}\left(\boldsymbol{\delta} \mathfrak{H}^{(l)} / \delta h_{-}\right), \quad l=n / 2, & n=2,4, \ldots\end{cases}
\end{aligned}
$$

in terms of energies/Hamiltonians $\mathfrak{H}^{(l)}=\int H^{(l)} d x$, where

$$
H^{(l)}=\frac{1}{2}(-1)^{l-1}\left(D_{x}^{l} h_{-}\right)^{2} \quad l=1,2, \ldots
$$

are local densities. Under the Hopf-Cole transformation (2.50), the hierarchy (2.68) is mapped to

$$
\begin{aligned}
& u_{t}=\mathscr{Q}^{n}\left(u_{x}\right), \quad n=1,2, \ldots \\
& =\left\{\begin{array}{lll}
\mathscr{S}\left(\delta \mathfrak{H}^{(l)} / \delta u\right), & l=(n+1) / 2, & n=1,3, \ldots \\
\mathscr{H}\left(\delta \mathfrak{H}^{(l)} / \delta u\right), & l=n / 2, & n=2,4, \ldots
\end{array}\right.
\end{aligned}
$$

where $\mathfrak{H}^{(l)}=\int H^{(l)} d x$ is given by the densities

$$
H^{(l)}=\frac{1}{2}(-1)^{l-1} \exp (2 v)\left(\left(D_{x}+u\right)^{l-1} u\right)^{2} \quad l=1,2, \ldots
$$

and where

$$
\mathscr{S}=D_{x} \exp (-2 v) D_{x}, \quad \mathscr{H}=\mathscr{Q} \mathscr{S}=D_{x} \exp (-v) D_{x} \exp (-v) D_{x}
$$

are a positive symmetric operator and a Hamiltonian operator, respectively. 
Corresponding to the potential form of Burgers' equation (2.57), the +1 flow in these two hierarchies (2.71) and (2.68) is given by the heat equation

$$
h_{-t}=h_{-x x}
$$

and Burgers' equation (up to a scaling of $u$ )

$$
u_{t}=u_{x x}+2 u u_{x} .
$$

Both of these equations are integrable and have a gradient structure

$$
h_{-t}=-\delta \mathfrak{H}^{(1)} / \delta h_{-}, \quad u_{t}=\mathscr{S}\left(\delta \mathfrak{H}^{(1)} / \delta u\right)
$$

in terms of the energy density

$$
H^{(1)}=\frac{1}{2} h_{-x}^{2}=\frac{1}{2} \exp (2 v) u^{2} .
$$

All of the odd flows have a similar structure.

The +2 flow in the hierarchies (2.71) and (2.68) is given by

$$
u_{t}=u_{x x x}+3\left(u u_{x x}+u_{x}^{2}+u^{2} u_{x}\right)
$$

and

$$
h_{-t}=h_{-x x x}
$$

which is the Airy equation. These two integrable equations have the Hamiltonian structure

$$
h_{-t}=-D_{x}\left(\delta \mathfrak{H}^{(1)} / \delta h_{-}\right), \quad u_{t}=\mathscr{S}\left(\delta \mathfrak{H}^{(1)} / \delta u\right)
$$

where the Hamiltonian is the same expression as the energy integral appearing in the +1 flow. There is a similar structure for all of the even flows.

Proposition 2. Each flow in both hierarchies (2.71) and (2.68) determines a null inelastic curve flow

$$
\vec{\gamma}_{t}=h_{+}^{(n)} e_{+}+h_{-}^{(n)} e_{-}, \quad n=1,2, \ldots
$$

whose components are given by

$$
\begin{aligned}
& h_{+}^{(n)}=h_{-}^{-1} D_{x}^{n}\left(h_{-}\right)=\left(D_{x}+u\right)^{n-1} u=\mathscr{R}^{n-1}\left(v_{x}\right), \\
& h_{-}^{(n)}=h_{-}=\exp \left(\int u d x\right)=\exp (v), \\
& n=1,2, \ldots
\end{aligned}
$$

where

$$
e_{+}=\vec{\gamma}_{x}, \quad e_{-}=\mathscr{N}\left(\vec{\gamma}_{x}\right)
$$

The expression

$$
u= \pm \eta\left(e_{ \pm}, e_{\mp x}\right)=-\eta\left(\mathscr{N}\left(\vec{\gamma}_{x}\right), \vec{\gamma}_{x x}\right)
$$

shows that $u$ and its $x$-derivatives are invariant under translations and boosts applied to the curve $\vec{\gamma}$ in the Minkowski plane, so thus the equations of motion (2.82) are invariant under the isometry group ISO $(1,1)$ of the Minkowski plane. 
These equations of motion (2.82) describe geometrical non-stretching motions of the curve. An interesting geometrical aspect of the motion emerges when the flow vector $\vec{\gamma}_{t}$ at each point $x$ on the curve is decomposed into complementary null components with respect to the light cone,

$$
\left(\vec{\gamma}_{t}\right)_{+}=\left(\left(D_{x}+u\right)^{n-1} u\right) e_{+}, \quad n=1,2, \ldots
$$

and

$$
\left(\vec{\gamma}_{t}\right)_{-}=h_{-} e_{-}
$$

where $\left(\vec{\gamma}_{t}\right)_{ \pm}$is a null vector parallel to $e_{ \pm}$. Now, from the frame structure equations (2.44), (2.46), (2.50)-(2.51), we have $\left(h_{-} e_{-}\right)_{x}=\left(h_{-x}-u h_{-}\right) e_{-}=0$ and $\left(h_{-} e_{-}\right)_{t}=\left(h_{-t}-\omega h_{-}\right) e_{-}=0$. Hence $h_{-} e_{-}$is a constant null vector, which lies on the opposite side of the light cone relative to the tangent vector of the curve, $\vec{\gamma}_{x}$, as shown by equation (2.84). This component (2.87) of the flow vector is the same for all of the flow equations (2.82). Moreover, going back to the original form of the frame structure equations (2.47)-(2.49), we see that this system has a consistent reduction if we put $h_{-}=0$, or correspondingly, $\left(\vec{\gamma}_{t}\right)_{-}=0$. Under this reduction, the main results given by theorems 3 and 4 continue to hold, as does the second half of Theorem 5, while only the Hopf-Cole transformation relating $u$ to $h_{-}$is lost.

The tangential component (2.86) of the flow vector determines a null inelastic curve flow by itself, which we denote

$$
\left(\vec{\gamma}_{t}\right)_{\|}=h_{+}^{(n)} T, \quad n=1,2, \ldots
$$

in terms of the tangent vector of the curve

$$
T=\vec{\gamma}_{x}
$$

with

$$
h_{+}^{(n)}=\left(D_{x}+u\right)^{n-1} u .
$$

All of these curve flows preserve the null condition $\eta\left(\vec{\gamma}_{x}, \vec{\gamma}_{x}\right)=0$ and exhibit invariance under the isometry group $I S O(1,1)$. Thus, each equation of motion (2.88) describes a geometric nonstretching motion of the curve.

The $n=1$ curve flow (2.88) corresponding to the heat equation (2.75), and equivalently to Burgers' equation (2.76) or its potential form (2.57), is given by

$$
\left(\vec{\gamma}_{t}\right)_{\|}=u T \text {. }
$$

Through the frame structure equation (2.44), we can express this equation of motion (2.91) in the simple form

$$
\left(\vec{\gamma}_{t}\right)_{\|}=\vec{\gamma}_{x x}, \quad \eta\left(\vec{\gamma}_{x}, \vec{\gamma}_{x}\right)=0
$$

which is a non-stretching heat map equation in the Minkowski plane.

Similarly, the $n=2$ curve flow (2.88) corresponding to the Airy equation (2.80), and equivalently to equations (2.79) and (2.61) obtained through the Hopf-Cole transformation, is given by

$$
\left(\vec{\gamma}_{t}\right)_{\|}=\left(u_{x}+u^{2}\right) T .
$$

This equation of motion (2.93) can be expressed entirely in terms of $x$-derivatives of $\vec{\gamma}_{x}$ by use of the frame structure equation (2.44), as follows. We have $u_{x} \vec{\gamma}_{x}=\left(u \vec{\gamma}_{x}\right)_{x}-u \vec{\gamma}_{x x}=\vec{\gamma}_{x x x}-u^{2} \vec{\gamma}_{x}$, which 
yields

$$
\left(\vec{\gamma}_{t}\right)_{\|}=\vec{\gamma}_{x x x}, \quad \eta\left(\vec{\gamma}_{x}, \vec{\gamma}_{x}\right)=0 .
$$

This curve flow is a non-stretching Airy map equation in the Minkowski plane.

\section{Timelike curve flows and integrable systems in $\mathbb{R}^{2,1}$}

Three-dimensional Minkowski space is a vector space $\mathbb{R}^{2,1}$ equipped with a Lorentz-signature $(-1,1,1)$ metric $\eta$ and a compatible volume form $\varepsilon$. A vector $\vec{v} \in \mathbb{R}^{2,1}$ is respectively timelike, spacelike, or null if its Minkowski norm $\eta(\vec{v}, \vec{v})$ is negative, positive, or zero. The set of all null vectors spans a 2-dimensional null surface through the origin in $\mathbb{R}^{2,1}$, called the lightcone.

Up to a sign, the volume form is determined from the metric by the relation

$$
\begin{array}{r}
\varepsilon(\vec{v}, \vec{w}, \vec{x})^{2}=\eta(\vec{v}, \vec{w})^{2} \eta(\vec{x}, \vec{x})+\eta(\vec{x}, \vec{v})^{2} \eta(\vec{w}, \vec{w})+\eta(\vec{w}, \vec{x})^{2} \eta(\vec{v}, \vec{v}) \\
-2 \eta(\vec{v}, \vec{w}) \eta(\vec{w}, \vec{x}) \eta(\vec{x}, \vec{v})-\eta(\vec{v}, \vec{v}) \eta(\vec{w}, \vec{w}) \eta(\vec{x}, \vec{x})
\end{array}
$$

holding for any vectors $\vec{v}, \vec{w}, \vec{x} \in \mathbb{R}^{2,1}$. Associated to the metric and the volume form is the Hodge dual operator $*$ defined by

$$
\eta(*(\vec{v} \wedge \vec{w}), \vec{x})=\varepsilon(\vec{v}, \vec{w}, \vec{x}) .
$$

This operator maps pairs of vectors into vectors, and thus it can be alternatively formulated as a Lorentzian version of the cross product defined by

$$
\vec{v} \times \vec{w}=*(\vec{v} \wedge \vec{w})
$$

which has the properties

$$
\vec{v} \times \vec{v}=0, \quad(\vec{v} \times \vec{w}) \times \vec{x}=\eta(\vec{w}, \vec{x}) \vec{v}-\eta(\vec{v}, \vec{x}) \vec{w}
$$

and

$$
\eta(\vec{v} \times \vec{w}, \vec{v})=\eta(\vec{v} \times \vec{w}, \vec{w})=0, \quad \eta(\vec{v} \times \vec{w}, \vec{v} \times \vec{w})=\eta(\vec{v}, \vec{w})^{2}-\eta(\vec{v}, \vec{v}) \eta(\vec{w}, \vec{w})
$$

holding for any vectors $\vec{v}, \vec{w}, \vec{x} \in \mathbb{R}^{2,1}$.

In inertial coordinates $X^{i}=\left(X^{0}, X^{1}, X^{2}\right)$, the Minkowski metric and the volume tensor respectively have the components

$$
\begin{gathered}
\eta_{i j}=\operatorname{diag}(-1,1,1)=\left\{\begin{array}{rl}
-1 & i=j=0 \\
1 & i=j=1,2 \\
0 & i \neq j
\end{array}\right. \\
\varepsilon_{i j k}=\operatorname{sgn}\left(\begin{array}{lll}
i & j & k \\
0 & 1 & 2
\end{array}\right)=\left\{\begin{aligned}
1 & (i, j, k)=(0,1,2), \text { cyclic } \\
-1 & (i, j, k)=(2,1,0), \text { cyclic } \\
0 & \text { otherwise }
\end{aligned}\right.
\end{gathered}
$$

while the Hodge dual operator $*$ and the cross product $\times$ are both given by the associated tensor $\varepsilon^{i}{ }_{j k}=\varepsilon_{l j k} \eta^{i l}$. Here $X^{0}$ is a timelike coordinate and $X^{1}, X^{2}$ are spacelike coordinates.

The isometries of 3-dimensional Minkowski space are given by the Poincare group $\operatorname{ISO}(2,1) \simeq$ $S O(2,1) \ltimes \mathbb{R}^{3}$, which comprises time and space translations, rotations, and boosts. 


\subsection{Timelike curves and parallel frames}

Let $\vec{\gamma}(x)=\left(X^{0}(x), X^{1}(x), X^{2}(x)\right)$ be a timelike curve in $\mathbb{R}^{2,1}$, with the tangent vector $\vec{\gamma}_{x}$ satisfying

$$
\eta\left(\vec{\gamma}_{x}, \vec{\gamma}_{x}\right)<0
$$

at every point $x$ on the curve. Hereafter we choose $x$ to be the proper-time arclength parameter defined by

$$
\left|\vec{\gamma}_{x}\right|=\sqrt{-\eta\left(\vec{\gamma}_{x}, \vec{\gamma}_{x}\right)}=1
$$

Then

$$
T=\vec{\gamma}_{x}, \quad \text { unit timelike tangent vector }
$$

satisfies

$$
\eta(T, T)=-1 .
$$

Hence the normal plane orthogonal to $T$ at each point on the curve is a spacelike (Euclidean) plane, $\mathbb{R}^{2}$.

We begin by introducing the Lorentzian analog of a Frenet frame for such curves [22]. The $x$ derivative of $T$ defines the principal normal vector $T_{x}$, which is spacelike since it lies in the normal plane, due to $\eta\left(T, T_{x}\right)=0$. Therefore

$$
N=\kappa^{-1} T_{x}, \quad \text { unit spacelike normal vector }
$$

satisfies

$$
\eta(T, N)=0, \quad \eta(N, N)=1
$$

where the function $\kappa(x)$ is given by

$$
\kappa=\eta\left(T_{x}, N\right)=\sqrt{\eta\left(T_{x}, T_{x}\right)}
$$

which is the Lorentzian curvature invariant of the curve. The cross product of $T$ and $T_{x}$ yields a vector which is orthogonal to both $T$ and $T_{x}$ from property (3.5). Since this vector $T \times T_{x}$ lies in the normal plane, it is spacelike. Hence

$$
B=\kappa^{-1} T \times T_{x}, \quad \text { unit spacelike bi-normal vector }
$$

satisfies

$$
\eta(B, T)=\eta(B, N)=0, \quad \eta(B, B)=1
$$

The triple of mutually orthogonal vectors

$$
\left(\begin{array}{l}
T \\
N \\
B
\end{array}\right)
$$

defines a Frenet frame for a timelike curve $\vec{\gamma}(x)$ in $\mathbb{R}^{2,1}$. Note that the orientation of this frame is

$$
\varepsilon(T, N, B)=1
$$

in terms of the volume form. 
The Frenet equations of this frame (3.17) are straightforward to derive. First, from equation (3.12), we have

$$
T_{x}=\kappa N .
$$

Next, the $x$-derivative of equation (3.13) yields $0=\eta\left(N_{x}, N\right)$ and $0=\eta\left(T_{x}, N\right)+\eta\left(T, N_{x}\right)=\kappa+$ $\eta\left(N_{x}, T\right)$, so thus we have

$$
N_{x}=\kappa T+\tau B
$$

for some function $\tau(x)$. To obtain an expression for $\tau$ in terms of $T$ and its $x$-derivatives, we substitute equations (3.12) and (3.15) into equation (3.20) and take its Minkowski inner product with $B$. This yields

$$
\tau=\eta\left(N_{x}, B\right)=\kappa^{-2} \eta\left(T \times T_{x}, T_{x x}\right)
$$

which is the Lorentzian torsion invariant of the curve. Last, from the $x$-derivative of equation (3.15), we find $B_{x}=T_{x} \times N+T \times N_{x}=\tau T \times B$ after using equations (3.19) and (3.20). Then using property (3.4), we have

$$
B_{x}=-\tau N
$$

Therefore, the Frenet equations are given by

$$
\left(\begin{array}{l}
T_{x} \\
N_{x} \\
B_{x}
\end{array}\right)=\left(\begin{array}{ccc}
0 & \kappa & 0 \\
\kappa & 0 & \tau \\
0 & -\tau & 0
\end{array}\right)\left(\begin{array}{l}
T \\
N \\
B
\end{array}\right)
$$

where $\left(\begin{array}{ccc}0 & \kappa & 0 \\ \kappa & 0 & \tau \\ 0 & -\tau & 0\end{array}\right) \in \mathfrak{s o}(2,1)$ is the Cartan matrix, which belongs to the Lie algebra $\mathfrak{s o}(2,1)$ of the $S O(2,1)$ group of rotation and boost isometries in $\mathbb{R}^{2,1}$.

A general frame for a timelike curve in $\mathbb{R}^{2,1}$ is related to this Frenet frame by the action of arbitrary $x$-dependent $S O(2,1)$ rotations and boosts applied to the vectors $(3.17)$. If the tangent vector $T$ is preserved as one of the frame vectors, then the resulting frame is given by applying a general $x$-dependent $S O(2)$ rotation on the normal vectors $N$ and $B$ in the spacelike normal plane of the curve. This yields

$$
\mathbf{E}=\left(\begin{array}{l}
e_{0} \\
e_{1} \\
e_{2}
\end{array}\right)=\left(\begin{array}{ccc}
1 & 0 & 0 \\
0 & \cos \theta & \sin \theta \\
0 & -\sin \theta & \cos \theta
\end{array}\right)\left(\begin{array}{l}
T \\
N \\
B
\end{array}\right)=\left(\begin{array}{c}
T \\
(\cos \theta) N+(\sin \theta) B \\
-(\sin \theta) N+(\cos \theta) B
\end{array}\right)
$$

where $\theta(x)$ is the rotation angle, and where the frame vectors satisfy the orthogonality relations

$$
\begin{gathered}
\eta\left(e_{0}, e_{0}\right)=-1, \quad \eta\left(e_{1}, e_{1}\right)=\eta\left(e_{2}, e_{2}\right)=1 \\
\eta\left(e_{0}, e_{1}\right)=\eta\left(e_{0}, e_{2}\right)=\eta\left(e_{1}, e_{2}\right)=0
\end{gathered}
$$

and the cross product relations

$$
e_{1} \times e_{2}=-e_{0}, \quad e_{0} \times e_{1}=e_{2}, \quad e_{0} \times e_{2}=-e_{1}
$$

which follow from $\varepsilon\left(e_{0}, e_{1}, e_{2}\right)=\varepsilon(T, N, B)=1$. 
We now use this gauge freedom to define a Lorentzian parallel frame by the geometrical condition that the $x$-derivative of both normal vectors $e_{1}$ and $e_{2}$ is parallel to the tangent vector $e_{0}$. Thus we require

$$
e_{1 x}=u_{1} e_{0}, \quad e_{2 x}=u_{2} e_{0}
$$

for some functions $u_{1}(x), u_{2}(x)$. This determines part of the Cartan matrix. To derive the remaining part, we need to work out $e_{0 x}$. By substituting equation (3.28) into the $x$-derivative of equations (3.25) and (3.26), we get $\eta\left(e_{0 x}, e_{1}\right)=u_{1}, \eta\left(e_{0 x}, e_{2}\right)=u_{2}$, and $\eta\left(e_{0 x}, e_{0}\right)=0$. These relations determine

$$
e_{0 x}=u_{1} e_{1}+u_{2} e_{2}
$$

Therefore, the Cartan matrix of a Lorentzian parallel frame for a timelike curve in $\mathbb{R}^{2,1}$ is given by

$$
\mathbf{E}_{x}=\mathbf{U E}
$$

where

$$
\mathbf{U}=\left(\begin{array}{ccc}
0 & u_{1} & u_{2} \\
u_{1} & 0 & 0 \\
u_{2} & 0 & 0
\end{array}\right) \in \mathfrak{s o}(2,1)
$$

This matrix belongs to the perp space of the stabilizer subalgebra $\mathfrak{s o}(2) \subset \mathfrak{s o}(2,1)$ of the frame vector $e_{0}$. In particular, there is a decomposition $\mathfrak{s o}(2,1)=\mathfrak{s o}(2) \oplus \mathfrak{s o}(2)_{\perp}$ as a symmetric Lie algebra, where $\mathfrak{s o}(2)$ is the subalgebra of rotations and $\mathfrak{s o}(2)_{\perp} \simeq \mathbb{R}^{2}$ is its perp space given by the span of the 1-dimensional subalgebras of boosts which act in two orthogonal planes containing $e_{0}$.

To determine the rotation angle $\theta(x)$ under which a Frenet frame is transformed to a parallel frame, we first need the inverse transformation

$$
\left(\begin{array}{l}
T \\
N \\
B
\end{array}\right)=\left(\begin{array}{c}
e_{0} \\
(\cos \theta) e_{1}-(\sin \theta) e_{2} \\
(\sin \theta) e_{1}+(\cos \theta) e_{2}
\end{array}\right) .
$$

Now, we take the $x$-derivative of the frame (3.24) and substitute the Frenet equations (3.23) followed by the inverse transformation (3.32), which yields

$$
\mathbf{E}_{x}=\left(\begin{array}{c}
(\kappa \cos \theta) e_{1}-(\kappa \sin \theta) e_{2} \\
(\kappa \cos \theta) e_{0}+\left(\theta_{x}+\tau\right) e_{2} \\
-(\kappa \sin \theta) e_{0}-\left(\theta_{x}+\tau\right) e_{1}
\end{array}\right)
$$

Thus the condition (3.30)-(3.31) can be achieved if (and only if)

$$
\theta_{x}=-\tau
$$

The resulting frame given by equations (3.24) and (3.34) thereby defines a parallel frame, where the components of its Cartan matrix are related to the curvature and torsion invariants $\kappa, \tau$ by

$$
u_{1}=\kappa \cos \theta=\kappa \cos \left(-\int \tau d x\right), \quad u_{2}=-\kappa \sin \theta=\kappa \sin \left(\int \tau d x\right) .
$$

These expressions are a Lorentzian counterpart of the well-known Hasimoto transformation in Euclidean space. 
A parallel frame is unique up to $x$-independent (rigid) rotations

$$
\theta \rightarrow \theta+\phi
$$

where $\phi$ is constant. Under these rotations, the tangent vector $e_{0}$ is preserved, while the normal vectors $e_{1}$ and $e_{2}$ are rigidly rotated

$$
\begin{aligned}
& e_{1} \rightarrow(\cos \phi) e_{1}+(\sin \phi) e_{2}, \\
& e_{2} \rightarrow-(\sin \phi) e_{1}+(\cos \phi) e_{2} .
\end{aligned}
$$

Any two parallel frames related by this transformation are gauge equivalent. The $S O(2)$ group of rigid rotations thereby defines the gauge (equivalence) group for parallel frames. Stated another way, a parallel frame is determined by a timelike curve only up to the action of this gauge group.

\subsection{Inelastic Flow Equations}

We now consider curve flows $\vec{\gamma}(t, x)$ that locally preserve both the timelike signature (3.8) of the curve and the proper time normalization (3.9) of the arclength parameter. Such flows are called inelastic and are specified by a flow vector

$$
\vec{\gamma}_{t}=h_{\|} e_{0}+h_{1} e_{1}+h_{2} e_{2}
$$

expressed in terms of a tangential component $h_{\|}$and a pair of normal components $h_{1}, h_{2}$ with respect to the frame vectors $e_{0}, e_{1}, e_{2}$.

The parallel frame will be carried by the flow, such that the orthogonality relations (3.25)-(3.26) are preserved. This implies that the $t$-derivative of the frame vectors $e_{0}, e_{1}, e_{2}$ is given by

$$
\begin{gathered}
e_{0 t}=\omega_{1} e_{1}+\omega_{2} e_{2}, \\
e_{1 t}=\omega_{1} e_{0}+\omega_{0} e_{2}, \quad e_{2 t}=\omega_{2} e_{0}-\omega_{0} e_{1} .
\end{gathered}
$$

We can write these equations in the form

$$
\mathbf{E}_{t}=\mathbf{W E}
$$

with the Cartan matrix

$$
\mathbf{W}=\left(\begin{array}{ccc}
0 & \omega_{1} & \omega_{2} \\
\omega_{1} & 0 & \omega_{0} \\
\omega_{2} & -\omega_{0} & 0
\end{array}\right) \in \mathfrak{s o}(2,1)
$$

which belongs to the Lie algebra of the $S O(2,1)$ group of rotation and boost isometries in $\mathbb{R}^{2,1}$.

The flow equations (3.41) and the Frenet equations (3.30) of the parallel frame are related by the compatibility condition $\partial_{t}\left(\mathbf{E}_{x}\right)=\partial_{x}\left(\mathbf{E}_{t}\right)$. This condition is equivalent to a zero curvature equation

$$
\mathbf{U}_{t}-\mathbf{W}_{x}+[\mathbf{U}, \mathbf{W}]=0
$$

relating the Cartan matrices $\mathbf{W}$ and $\mathbf{U}$. After substituting these matrices (3.31) and (3.42) into equation (3.43), we obtain

$$
\begin{gathered}
u_{1 t}=\omega_{1 x}+u_{2} \omega_{0}, \quad u_{2 t}=\omega_{2 x}-u_{1} \omega_{0} \\
\omega_{0 x}=u_{1} \omega_{2}-\omega_{1} u_{2} .
\end{gathered}
$$


Likewise, the flow vector (3.38) and the tangent vector (3.10) of the curve are related by the compatibility condition $\partial_{x}\left(\vec{\gamma}_{t}\right)=\partial_{t}\left(\vec{\gamma}_{x}\right)$. By writing

$$
\mathbf{H}=\left(\begin{array}{l}
h_{\|} \\
h_{1} \\
h_{2}
\end{array}\right) \in \mathbb{R}^{3}, \quad \mathbf{A}=\left(\begin{array}{l}
1 \\
0 \\
0
\end{array}\right) \in \mathbb{R}^{3},
$$

we have $\vec{\gamma}_{x}=\mathbf{A}^{\mathrm{T}} \mathbf{E}$ and $\vec{\gamma}_{t}=\mathbf{H}^{\mathrm{T}} \mathbf{E}$. Then the compatibility condition becomes

$$
\mathbf{H}_{x}+\mathbf{U}^{\mathrm{T}} \mathbf{H}=\mathbf{W}^{\mathrm{T}} \mathbf{A}
$$

relating $\mathbf{W}$ to $\mathbf{U}$ and $\mathbf{H}$. After we substitute the matrices (3.31) and (3.42) along with the vectors (3.46) into equation (3.47), we find that its tangential and normal components yield

$$
\begin{gathered}
h_{\| x}=-h_{1} u_{1}-h_{2} u_{2}, \\
\omega_{1}=h_{1 x}+u_{1} h_{\|}, \quad \omega_{2}=h_{2 x}+u_{2} h_{\|} .
\end{gathered}
$$

These compatibility equations (3.44), (3.45), (3.48), (3.49) are the Cartan structure equations of the parallel frame. They describe all inelastic timelike curve flows $\vec{\gamma}(t, x)$ in $\mathbb{R}^{2,1}$.

\subsection{U(1)-invariant formalism}

The gauge group for parallel frames consists of rigid $S O(2)$ rotations (3.37) acting on the normal vectors. Under the action of this group, the components of the Cartan matrix (3.31) along the curve are transformed by

$$
\begin{aligned}
& u_{1} \rightarrow u_{1} \cos \phi+u_{2} \sin \phi=\kappa \cos (\theta+\phi), \\
& u_{2} \rightarrow-u_{1} \sin \phi+u_{2} \cos \phi=-\kappa \sin (\theta+\phi) .
\end{aligned}
$$

Similarly, the components of the Cartan matrix (3.42) along the flow are transformed by

$$
\begin{gathered}
\omega_{0} \rightarrow \omega_{0}, \\
\omega_{1} \rightarrow \omega_{1} \cos \phi+\omega_{2} \sin \phi, \\
\omega_{2} \rightarrow-\omega_{1} \sin \phi+\omega_{2} \cos \phi .
\end{gathered}
$$

Since the flow vector (3.38) is gauge invariant, its normal and tangential components in a parallel frame are transformed by

$$
\begin{gathered}
h_{\|} \rightarrow h_{\|}, \\
h_{1} \rightarrow h_{1} \cos \phi+h_{2} \sin \phi, \\
h_{2} \rightarrow-h_{1} \sin \phi+h_{2} \cos \phi .
\end{gathered}
$$

Hence it is natural to introduce a complex formalism

$$
u=u_{1}+i u_{2}=\kappa e^{-i \theta}=\kappa \exp \left(i \int \tau d x\right), \quad \omega=\omega_{1}+i \omega_{2}, \quad h_{\perp}=h_{1}+i h_{2}
$$

in which the rigid rotations (3.50), (3.52), (3.54) become phase rotations

$$
u \rightarrow u \exp (-i \phi), \quad \omega \rightarrow \omega \exp (-i \phi), \quad h_{\perp} \rightarrow h_{\perp} \exp (-i \phi) .
$$


Now, we can write the system (3.44), (3.45), (3.48), (3.49) in a $U(1)$-invariant form:

$$
\begin{gathered}
u_{t}=\omega_{x}-i u \omega_{0}, \\
\omega_{0 x}=\operatorname{Im}(\bar{u} \omega), \\
h_{\| x}=-\operatorname{Re}\left(\bar{u} h_{\perp}\right), \\
\omega=h_{\perp x}+u h_{\|} .
\end{gathered}
$$

Since $u$ is determined by the curve only up to rigid $U(1)$ phase rotations (3.50), this complex variable $u$ is a Hasimoto variable which geometrically represents a $U(1)$-covariant of the curve, in contrast to the invariants $\kappa, \tau$ which are determined uniquely by the curve.

This system (3.57)-(3.60) has the following operator formulation which encodes a triple of $U(1)$-invariant Hamiltonian operators.

Theorem 6. For timelike inelastic curve flows in $\mathbb{R}^{2,1}$, the curve covariant $u$ satisfies the $U(1)$ invariant system

$$
\begin{aligned}
& u_{t}=D_{x} \omega-i u D_{x}^{-1} \operatorname{Im}(\omega \bar{u})=\mathscr{H}(\omega) \\
& \omega=D_{x} h_{\perp}-u D_{x}^{-1} \operatorname{Re}\left(\bar{u} h_{\perp}\right)=\mathscr{J}\left(h_{\perp}\right)
\end{aligned}
$$

where

$$
\begin{aligned}
\mathscr{H} & =D_{x}+i u D_{x}^{-1} \operatorname{Im}(u \mathscr{C}) \\
\mathscr{J} & =D_{x}-u D_{x}^{-1} \operatorname{Re}(u \mathscr{C})
\end{aligned}
$$

are a pair of compatible, $U(1)$-invariant Hamiltonian and symplectic operators, and $\mathscr{C}$ denotes the complex conjugation operator. Moreover, the operators $\mathscr{H}$ and $\mathscr{J}$ are related by

$$
\mathscr{H} \mathscr{I}^{-1}=-\mathscr{I} \mathscr{J}, \quad \mathscr{J} \mathscr{I}=-\mathscr{I}^{-1} \mathscr{H}, \quad \mathscr{I}=-i
$$

where $\mathscr{I}$ is a Hamiltonian operator compatible with $\mathscr{H}$ and $\mathscr{J}$. Composition of these operators yields the $U(1)$-invariant evolution equation

$$
u_{t}=-\mathscr{R}^{2}\left(h_{\perp}\right)
$$

for $u$ in terms of the normal component $h_{\perp}$ of the flow, where

$$
\mathscr{R}=\mathscr{H} \mathscr{I}^{-1}=-\mathscr{I} \mathscr{J}=i\left(D_{x}-u D_{x}^{-1} \operatorname{Re}(u \mathscr{C})\right)
$$

is a hereditary recursion operator.

This theorem is a counterpart of a similar result $[4,18]$ for inelastic curve flows in $\mathbb{R}^{3}$ and can be proved by the same methods. In particular, compared to the Euclidean case, there is only a change in the sign of the nonlocal term in both operators $\mathscr{H}$ and $\mathscr{J}$.

An operator $\mathscr{D}$ here is Hamiltonian iff it defines an associated Poisson bracket

$$
\{\mathfrak{H}, \mathfrak{G}\}_{\mathscr{D}}=\int \operatorname{Re}((\delta \mathfrak{H} / \delta u) \mathscr{D}(\delta \mathfrak{G} / \delta \bar{u})) d x
$$

obeying skew-symmetry $\{\mathfrak{H}, \mathfrak{G}\}_{\mathscr{D}}+\{\mathfrak{G}, \mathfrak{H}\}_{\mathscr{D}}=0$ and the Jacobi identity $\left\{\{\mathfrak{H}, \mathfrak{G}\}_{\mathscr{D}}, \mathfrak{F}\right\}_{\mathscr{D}}+$ cyclic $=0$ for all functionals $\mathfrak{H}, \mathfrak{G}, \mathfrak{F}$ on the $x$-jet space $J^{\infty}$ of the variables $u$ and $\bar{u}$, where $\delta / \delta u$ and 
$\delta / \delta \bar{u}$ denote the standard Euler operators. The formal inverse of a Hamiltonian operator defines a symplectic operator. Compatibility of two Hamiltonian operators $\mathscr{D}_{1}$ and $\mathscr{D}_{2}$ is the statement that every linear combination $c_{1} \mathscr{D}_{1}+c_{2} \mathscr{D}_{2}$ is a Hamiltonian operator.

Due to a general theorem of Magri [17], the $U(1)$ invariance of the pair of compatible Hamiltonian operators $\mathscr{H}$ and $\mathscr{I}$ can be used to derive a hierarchy of flows starting from a root flow $u_{t}=-i u$ corresponding to the generator of $U(1)$ phase-rotations on $u$. This leads to the following main result.

Theorem 7. There is a hierarchy of integrable tri-Hamiltonian flows on $u(t, x)$ given by

$$
\begin{aligned}
u_{t}=\mathscr{R}^{n+1}(-i u) & =\mathscr{I}\left(\delta \mathfrak{H}^{(n+1)} / \delta \bar{u}\right)=\mathscr{H}\left(\delta \mathfrak{H}^{(n)} / \delta \bar{u}\right), \quad n=0,1,2, \ldots \\
& =\mathscr{E}\left(\delta \mathfrak{H}^{(n-1)} / \delta \bar{u}\right), \quad n=1,2, \ldots
\end{aligned}
$$

(called the $+n$ flow) in terms of Hamiltonians $\mathfrak{H}^{(n)}=\int H^{(n)} d x$ where

$$
\begin{aligned}
& \mathscr{I}=-i, \quad \mathscr{H}=D_{x}+i u D_{x}^{-1} \operatorname{Im}(u \mathscr{C}), \\
& \mathscr{E}=\mathscr{R} \mathscr{H}=i\left(D_{x}{ }^{2}-|u|^{2}+u D_{x}^{-1} \operatorname{Re}\left(u_{x} \mathscr{C}\right)+i u_{x} D_{x}^{-1} \operatorname{Im}(u \mathscr{C})\right)
\end{aligned}
$$

are compatible Hamiltonian operators, and where

$$
H^{(n)}=2(1+n)^{-1} D_{x}^{-1} \operatorname{Im}\left(\bar{u}(i \mathscr{H})^{n+1} u\right), \quad n=0,1,2, \ldots
$$

are local Hamiltonian densities.

The expression for the Hamiltonians (3.71) in this theorem arises from a scaling formula derived in Ref. [1].

The +1 flow in the hierarchy (3.69) is explicitly given by

$$
u_{t}=i\left(u_{x x}-\frac{1}{2}|u|^{2} u\right)
$$

which is the defocusing NLS equation. It has the explicit tri-Hamiltonian structure

$$
u_{t}=\mathscr{I}\left(\delta \mathfrak{H}^{(2)} / \delta \bar{u}\right)=\mathscr{H}\left(\delta \mathfrak{H}^{(1)} / \delta \bar{u}\right)=\mathscr{E}\left(\delta \mathfrak{H}^{(0)} / \delta \bar{u}\right)
$$

in terms of the Hamiltonian densities

$$
H^{(0)}=|u|^{2}, \quad H^{(1)}=\operatorname{Im}\left(u \bar{u}_{x}\right), \quad H^{(2)}=\left|u_{x}\right|^{2}+\frac{1}{4}|u|^{4}
$$

(modulo trivial densities given by total $x$-derivatives). Similarly, the +2 flow in the hierarchy (3.69) is given by

$$
u_{t}=u_{x x x}-\frac{3}{2}|u|^{2} u_{x}
$$

which is the complex defocusing $\mathrm{mKdV}$ equation. It has the tri-Hamiltonian structure

$$
u_{t}=\mathscr{I}\left(\delta \mathfrak{H}^{(3)} / \delta \bar{u}\right)=\mathscr{H}\left(\delta \mathfrak{H}^{(2)} / \delta \bar{u}\right)=\mathscr{E}\left(\delta \mathfrak{H}^{(1)} / \delta \bar{u}\right)
$$

where $\mathfrak{H}^{(1)}$ and $\mathfrak{H}^{(2)}$ are given by the Hamiltonian densities (3.74), while $\mathfrak{H}^{(3)}$ is given by the Hamiltonian density

$$
H^{(3)}=\operatorname{Im}\left(u_{x} \bar{u}_{x x}\right)+\frac{3}{4}|u|^{2} \operatorname{Im}\left(u \bar{u}_{x}\right)
$$

(modulo trivial densities given by total $x$-derivatives). 
The entire hierarchy (3.69) of flows corresponds to a hierarchy of commuting vector fields

$$
\mathrm{X}^{(n)}=\mathscr{R}^{n}(-i u) \partial_{u}, \quad n=0,1,2, \ldots
$$

with the root vector field being the generator of phase rotations $\mathrm{X}^{(0)}=-i u \partial_{u}$. In this setting, a vector field is Hamiltonian iff its prolongation on the $x$-jet space $J^{\infty}$ of $u$ and $\bar{u}$ satisfies $\operatorname{prX\mathfrak {G}}=$ $\{\mathfrak{G}, \mathfrak{H}\}_{\mathscr{D}}$ for some functional $\mathfrak{H}$, where $\mathfrak{G}$ is an arbitrary functional, and $\mathscr{D}$ is a given Hamiltonian operator. As shown by Theorem 2, all of the vector fields in this hierarchy are bi-Hamiltonian, with $\operatorname{prX}^{(n)} \mathfrak{G}=\left\{\mathfrak{G}, \mathfrak{H}^{(n)}\right\}_{\mathscr{H}}=\left\{\mathfrak{G}, \mathfrak{H}^{(n+1)}\right\}_{\mathscr{I}}$ for $n \geq 0$, where $\mathfrak{H}^{(n)}=\int H^{(n)} d x$ is the functional with the Hamiltonian density (3.71), while each vector field for $n \geq 1$ is also tri-Hamiltonian, $\operatorname{prX}^{(n)} \mathfrak{G}=$ $\left\{\mathfrak{G}, \mathfrak{H}^{(n-1)}\right\}_{\mathscr{E}}$. Since the entire hierarchy is commuting, every Hamiltonian vector field (3.78) is the generator of a symmetry for the defocusing NLS equation (3.72) as well as the defocusing $\mathrm{mKdV}$ equation (3.75), and every associated Hamiltonian (3.71) is a conserved density for both of these equations.

Proposition 3. Each tri-Hamiltonian flow in the hierarchy (3.69) can be written in the form (3.66) as given by

$$
h_{\perp}=-\mathscr{R}^{n-1}(-i u), \quad n=1,2, \ldots
$$

Consequently, the $+n$ flow for $n \geq 1$ determines an inelastic timelike curve flow

$$
\vec{\gamma}_{t}=h_{\|}^{(n)} e_{0}+h_{1}^{(n)} e_{1}+h_{2}^{(n)} e_{2} \quad n=1,2, \ldots
$$

whose tangential and normal components are given by

$$
h_{\|}^{(n)}=\frac{1}{2}(n-1) H^{(n-2)}, \quad h_{1}^{(n)}+i h_{2}^{(n)}=-\mathscr{R}^{n-1}(-i u), \quad n=1,2, \ldots
$$

To write these equation of motions (3.80) in an explicit form, it is convenient to introduce a complex parallel-frame notation:

$$
e_{\|}=e_{0}=T, \quad e_{\perp}=e_{1}+i e_{2}=e^{-i \theta}(N+i B)
$$

satisfying

$$
e_{\|} \times e_{\perp}=-i e_{\perp}, \quad e_{\perp} \times \bar{e}_{\perp}=i 2 e_{\|}
$$

and

$$
\eta\left(e_{\|}, e_{\|}\right)=-1, \quad \eta\left(e_{\perp}, \bar{e}_{\perp}\right)=2, \quad \eta\left(e_{\|}, e_{\perp}\right)=\eta\left(e_{\perp}, e_{\perp}\right)=0 .
$$

The action of the gauge group (3.37) on these frame vectors consists of rigid $U(1)$ phase rotations

$$
e_{\|} \rightarrow e_{\|}, \quad e_{\perp} \rightarrow e^{-i \phi} e_{\perp}
$$

where $\phi$ is constant. 
The Frenet equations (3.30) of the underlying parallel frame become

$$
e_{\| x}=\operatorname{Re}\left(\bar{u} e_{\perp}\right), \quad e_{\perp x}=u e_{\|},
$$

while the flow equations (3.41) are similarly given by

$$
e_{\| t}=\operatorname{Re}\left(\bar{\omega} e_{\perp}\right), \quad e_{\perp t}=\omega e_{\|}-i \omega_{0} e_{\perp}
$$

The equation of motions (3.80) for the curve now take the form

$$
\vec{\gamma}_{t}=h_{\|}^{(n)} e_{\|}+\operatorname{Re}\left(\bar{h}_{\perp}^{(n)} e_{\perp}\right), \quad n=1,2, \ldots
$$

where the tangential component $h_{\|}^{(n)}$ and the normal component $h_{\perp}^{(n)}=h_{1}^{(n)}+i h_{2}^{(n)}$ are functions of the curve covariant $u$, the complex conjugate covariant $\bar{u}$, and their $x$-derivatives, as given by the expressions (3.81). Since $u \rightarrow e^{-i \phi} u$ and $\bar{u} \rightarrow e^{i \phi} \bar{u}$ under the gauge group (3.85), the tangential component is gauge invariant while the normal component is gauge equivariant,

$$
h_{\|}^{(n)} \rightarrow h_{\|}^{(n)}, \quad h_{\perp}^{(n)} \rightarrow e^{-i \phi} h_{\perp}^{(n)} .
$$

Consequently, each equation of motion (3.38) is invariant under the isometry group $\operatorname{ISO}(2,1)$ of $\mathbb{R}^{2,1}$ and thus describes a geometric non-stretching motion of the curve.

The curve flow $n=1$ corresponding to the defocusing NLS equation (3.72) is determined by the components

$$
h_{\|}^{(1)}=0, \quad h_{\perp}^{(1)}=i u
$$

Substituting these expressions into the $n=1$ equation of motion (3.38), we have $\vec{\gamma}_{t}=\operatorname{Re}\left(-i \bar{u} e_{\perp}\right)=$ $\operatorname{Re}\left(\bar{u} e_{\|} \times e_{\perp}\right)=e_{\|} \times e_{\| x}$ by using the cross product identity (3.83) and the Frenet equations (3.86). Thus we obtain

$$
\vec{\gamma}_{t}=T \times T_{x}=\vec{\gamma}_{x} \times \vec{\gamma}_{x x}, \quad \eta\left(\vec{\gamma}_{x}, \vec{\gamma}_{x}\right)=-1
$$

which is a timelike version of the vortex filament equation in Minkowski space $\mathbb{R}^{2,1}$.

The curve flow $n=2$ corresponding to the defocusing $\mathrm{mKdV}$ equation (3.75) has the components

$$
h_{\|}^{(2)}=\frac{1}{2}|u|^{2}, \quad h_{\perp}^{(2)}=-u_{x}
$$

Substituting these expressions into the $n=2$ equation of motion (3.38), we find $\vec{\gamma}_{t}=\operatorname{Re}\left(-\bar{u}_{x} e_{\perp}\right)+$ $\frac{1}{2}|u|^{2} e_{\|}$. We simplify the term $\operatorname{Re}\left(-\bar{u}_{x} e_{\perp}\right)=-\operatorname{Re}\left(\bar{u} e_{\perp}\right)_{x}+\operatorname{Re}\left(\bar{u} e_{\perp x}\right)=-e_{\| x x}+|u|^{2} e_{\|}$by using the Frenet equations (3.86). Next we note $\eta\left(e_{\| x}, e_{\| x}\right)=\eta\left(\operatorname{Re}\left(\bar{u} e_{\perp}\right), \operatorname{Re}\left(\bar{u} e_{\perp}\right)\right)=\frac{1}{2} \eta\left(\bar{u} e_{\perp}, u \bar{e}_{\perp}\right)=|u|^{2}$ which follows from the relations (3.84). This yields $\vec{\gamma}_{t}=-e_{\| x x}+\frac{3}{2}|u|^{2} e_{\|}$, and thus we obtain

$$
\vec{\gamma}_{t}=-T_{x x}+\frac{3}{2} \eta\left(T_{x}, T_{x}\right) T=-\vec{\gamma}_{x x x}+\frac{3}{2} \eta\left(\vec{\gamma}_{x x}, \vec{\gamma}_{x x}\right) \vec{\gamma}_{x}, \quad \eta\left(\vec{\gamma}_{x}, \vec{\gamma}_{x}\right)=-1
$$

which is a timelike version of the non-stretching mKdV map equation [2] in Minkowski space $\mathbb{R}^{2,1}$. 


\section{Spacelike curve flows and integrable systems in $\mathbb{R}^{2,1}$}

In 3-dimensional Minkowski space $\mathbb{R}^{2,1}$, let $\vec{\gamma}(x)=\left(X^{0}(x), X^{1}(x), X^{2}(x)\right)$ be a spacelike curve, with the tangent vector $\vec{\gamma}_{x}$ satisfying

$$
\eta\left(\vec{\gamma}_{x}, \vec{\gamma}_{x}\right)>0
$$

at every point $x$ on the curve. Hereafter we choose $x$ to be the proper-distance arclength parameter defined by

$$
\left|\vec{\gamma}_{x}\right|=\sqrt{\eta\left(\vec{\gamma}_{x}, \vec{\gamma}_{x}\right)}=1
$$

Then

$$
T=\vec{\gamma}_{x}, \quad \text { unit spacelike tangent vector }
$$

satisfies

$$
\eta(T, T)=1
$$

Hence the normal plane orthogonal to $T$ at each point on the curve is a Minkowski plane, $\mathbb{R}^{1,1}$. The $x$-derivative of $T$ defines the principal normal vector $T_{x}$ which lies in this plane, due to $\eta\left(T, T_{x}\right)=0$. Thus $T_{x}$ can be either timelike, spacelike, or null. The case when $T_{x}$ is non-null is similar to the cases of timelike/spacelike curve flows in 2-dimensional Minkowski space discussed in Sec. 2.1. In contrast, the case when $T_{x}$ is null has no counterpart in 2-dimensional Minkowski space.

We will consider the non-null case first and discuss the null case afterward.

\subsection{Spacelike curve flows with a non-null normal vector}

We begin by introducing the Lorentzian analog of a Frenet frame for spacelike curves whose principal normal vector $T_{x}$ is assumed to be non-null

$$
\eta\left(T_{x}, T_{x}\right) \neq 0
$$

at every point on the curve. In this case,

$$
N=\kappa^{-1} T_{x}, \quad \text { unit timelike/spacelike normal vector }
$$

satisfies

$$
\eta(T, N)=0, \quad \eta(N, N)=\mp 1
$$

where the function $\kappa(x)$ is given by

$$
\kappa=\mp \eta\left(T_{x}, N\right)=\sqrt{\mp \eta\left(T_{x}, T_{x}\right)}
$$

which is the Lorentzian curvature invariant of the curve. As shown by property (3.5), the cross product of $T$ and $T_{x}$ yields a vector which is orthogonal to both $T$ and $T_{x}$ and has the norm $\eta(T \times$ 
$\left.T_{x}, T \times T_{x}\right)=-\eta\left(T_{x}, T_{x}\right)$ which is opposite in sign to the norm of $T_{x}$. Hence

$$
B=\kappa^{-1} T \times T_{x}, \quad \text { unit spacelike/timelike bi-normal vector }
$$

satisfies

$$
\eta(B, T)=\eta(B, N)=0, \quad \eta(B, B)= \pm 1 .
$$

The triple of mutually orthogonal vectors

$$
\left(\begin{array}{l}
T \\
N \\
B
\end{array}\right)
$$

thereby defines a Frenet frame for a spacelike curve $\vec{\gamma}(x)$ with a non-null principal normal in $\mathbb{R}^{2,1}$. This frame has the orientation

$$
\varepsilon(T, N, B)= \pm 1
$$

in terms of the volume form.

The Frenet equations of this frame (4.11) can be derived similarly to the case of timelike curves in Sec. 3.1, with some changes of signs. First, from equation (4.6),

$$
T_{x}=\kappa N
$$

is the same. Next, from the $x$-derivative of equation (4.7), we have

$$
N_{x}= \pm \kappa T+\tau B
$$

for some function $\tau(x)$. An expression for $\tau$ can be obtained in terms of $T$ and its $x$-derivatives by use of equations (4.6) and (4.9). This yields

$$
\tau= \pm \eta\left(N_{x}, B\right)= \pm \kappa^{-2} \eta\left(T \times T_{x}, T_{x x}\right)
$$

which is the Lorentzian torsion invariant of the curve. Last, from the $x$-derivative of equation (4.9) combined with equations (4.13), (4.14), and property (3.4), we have

$$
B_{x}=\tau N
$$

which has changed in sign.

Therefore, the Frenet equations are given by

$$
\left(\begin{array}{l}
T_{x} \\
N_{x} \\
B_{x}
\end{array}\right)=\left(\begin{array}{ccc}
0 & \kappa & 0 \\
\pm \kappa & 0 & \tau \\
0 & \tau & 0
\end{array}\right)\left(\begin{array}{l}
T \\
N \\
B
\end{array}\right)
$$

where $\left(\begin{array}{ccc}0 & \kappa & 0 \\ \pm \kappa & 0 & \tau \\ 0 & \tau & 0\end{array}\right) \in \mathfrak{s o}(2,1)$ is the Cartan matrix, which belongs to the Lie algebra $\mathfrak{s o}(2,1)$ of the $S O(2,1)$ group of rotation and boost isometries in $\mathbb{R}^{2,1}$.

A general frame for a spacelike curve in $\mathbb{R}^{2,1}$ is related to this Frenet frame by the action of arbitrary $x$-dependent $S O(2,1)$ rotations and boosts applied to the vectors $(4.11)$. If the tangent 
vector $T$ is preserved as one of the frame vectors, then the resulting frame is given by applying a general $x$-dependent $S O(1,1)$ boost on the normal vectors $N$ and $B$ in the $\mathbb{R}^{1,1}$ normal plane of the curve. Geometrically, this transformation on the Frenet frame is a hyperbolic rotation, yielding

$$
\mathbf{E}=\left(\begin{array}{l}
e_{\|} \\
e_{1} \\
e_{2}
\end{array}\right)=\left(\begin{array}{ccc}
1 & 0 & 0 \\
0 & \cosh \theta & -\sinh \theta \\
0 & -\sinh \theta & \cosh \theta
\end{array}\right)\left(\begin{array}{l}
T \\
N \\
B
\end{array}\right)=\left(\begin{array}{c}
T \\
(\cosh \theta) N-(\sinh \theta) B \\
-(\sinh \theta) N+(\cosh \theta) B
\end{array}\right)
$$

where $\theta(x)$ is the hyperbolic rotation angle, and where the frame vectors satisfy the orthogonality relations

$$
\begin{gathered}
\eta\left(e_{\|}, e_{\|}\right)=1, \quad \eta\left(e_{1}, e_{1}\right)=-\eta\left(e_{2}, e_{2}\right)=\mp 1 \\
\eta\left(e_{\|}, e_{1}\right)=\eta\left(e_{\|}, e_{2}\right)=\eta\left(e_{1}, e_{2}\right)=0
\end{gathered}
$$

and the cross product relations

$$
e_{1} \times e_{2}= \pm e_{\|}, \quad e_{\|} \times e_{1}=e_{2}, \quad e_{\|} \times e_{2}=e_{1}
$$

which follow from $\varepsilon\left(e_{0}, e_{1}, e_{2}\right)=\varepsilon(T, N, B)= \pm 1$.

Similarly to the case of timelike curves, this gauge freedom can be used to define a Lorentzian parallel frame by the geometrical condition that the $x$-derivative of both normal vectors $e_{1}$ and $e_{2}$ is parallel to the tangent vector $e_{\|}$. Thus we require

$$
e_{1 x}=u_{1} e_{\|}, \quad e_{2 x}=u_{2} e_{\|}
$$

for some functions $u_{1}(x), u_{2}(x)$. To obtain $e_{\| x}$, we substitute equation (4.22) into the $x$-derivative of equations (4.19) and (4.20). This yields the relations $\eta\left(e_{\| x}, e_{1}\right)=-u_{1}, \eta\left(e_{\| x}, e_{2}\right)=-u_{2}$, and $\eta\left(e_{\| x}, e_{\|}\right)=0$, which determine

$$
e_{\| x}= \pm\left(u_{1} e_{1}-u_{2} e_{2}\right)
$$

Therefore, the Cartan matrix of a Lorentzian parallel frame for a spacelike curve with a non-null principal normal in $\mathbb{R}^{2,1}$ is given by

$$
\mathbf{E}_{x}=\mathbf{U E}
$$

where

$$
\mathbf{U}=\left(\begin{array}{ccc}
0 & \pm u_{1} \mp u_{2} \\
u_{1} & 0 & 0 \\
u_{2} & 0 & 0
\end{array}\right) \in \mathfrak{s o}(2,1)
$$

This matrix belongs to the perp space of the stabilizer subalgebra $\mathfrak{s o}(1,1) \subset \mathfrak{s o}(2,1)$ of the frame vector $e_{\|}$. The perp space $\mathfrak{s o}(1,1)_{\perp} \simeq \mathbb{R}^{1,1}$ is given by the span of the rotation subalgebra $\mathfrak{s o}(2)$ and another boost subalgebra $\mathfrak{s o}(1,1)$ which act in two orthogonal planes (one spacelike and one timelike) containing $e_{\|}$. In particular, $\mathfrak{s o}(1,1) \oplus \mathfrak{s o}(1,1)_{\perp}=\mathfrak{s o}(2,1)$ is a symmetric Lie algebra decomposition. 
To determine the hyperbolic rotation angle $\theta(x)$ under which a Frenet frame is transformed to a parallel frame, we first need the inverse transformation

$$
\left(\begin{array}{c}
T \\
N \\
B
\end{array}\right)=\left(\begin{array}{c}
e_{\|} \\
(\cosh \theta) e_{1}+(\sinh \theta) e_{2} \\
(\sinh \theta) e_{1}+(\cosh \theta) e_{2}
\end{array}\right)
$$

Now, we take the $x$-derivative of the frame (4.18) and substitute the Frenet equations (4.17) followed by the inverse transformation (4.26), which yields

$$
\mathbf{E}_{x}=\left(\begin{array}{c}
(\kappa \cosh \theta) e_{1}+(\kappa \sinh \theta) e_{2} \\
\pm(\kappa \cosh \theta) e_{\|}-\left(\theta_{x}-\tau\right) e_{2} \\
\mp(\kappa \sinh \theta) e_{\|}-\left(\theta_{x}-\tau\right) e_{1}
\end{array}\right)
$$

Thus the condition (4.24)-(4.25) can be achieved if (and only if)

$$
\theta_{x}=\tau
$$

The resulting frame given by equations (4.18) and (4.28) thereby defines a parallel frame, where the components of its Cartan matrix are related to the curvature and torsion invariants $\kappa, \tau$ by

$$
u_{1}= \pm \kappa \cosh \theta= \pm \kappa \cosh \left(\int \tau d x\right), \quad u_{2}=\mp \kappa \sinh \theta=\mp \kappa \sinh \left(\int \tau d x\right) .
$$

These expressions are a Lorentzian counterpart of the well-known Hasimoto transformation [15] in Euclidean space.

A parallel frame is unique up to $x$-independent (rigid) hyperbolic rotations

$$
\theta \rightarrow \theta+\phi
$$

where $\phi$ is constant. Under these transformations, the tangent vector $e_{\|}$is preserved, while the normal vectors $e_{1}$ and $e_{2}$ are rigidly rotated

$$
\begin{aligned}
& e_{1} \rightarrow(\cosh \phi) e_{1}-(\sinh \phi) e_{2}, \\
& e_{2} \rightarrow-(\sinh \phi) e_{1}+(\cosh \phi) e_{2} .
\end{aligned}
$$

Any two parallel frames related by this hyperbolic rotation are gauge equivalent. The $S O(1,1)$ group of rigid hyperbolic rotations thereby defines the gauge (equivalence) group for parallel frames. Stated another way, a parallel frame for a spacelike curve with a non-null principal normal is determined only up to the action of this gauge group.

\subsubsection{Inelastic Flow Equations}

We now consider spacelike inelastic curve flows $\vec{\gamma}(t, x)$ that locally preserve the proper distance normalization (3.9) of the arclength parameter and the non-null signature (4.5) of the principal normal. Such flows are specified by a flow vector

$$
\vec{\gamma}_{t}=h_{\|} e_{\|}+h_{1} e_{1}+h_{2} e_{2}
$$

expressed in terms of a tangential component $h_{\|}$and a pair of normal components $h_{1}, h_{2}$ with respect to the frame vectors $e_{\|}, e_{1}, e_{2}$. 
The parallel frame will be carried by the flow, such that the orthogonality relations (4.19)-(4.20) are preserved. This implies that the $t$-derivative of the frame vectors $e_{\|}, e_{1}, e_{2}$ is given by

$$
\begin{gathered}
e_{\| t}= \pm\left(\omega_{1} e_{1}-\omega_{2} e_{2}\right), \\
e_{1 t}=\omega_{1} e_{\|}+\omega_{0} e_{2}, \quad e_{2 t}=\omega_{2} e_{\|}+\omega_{0} e_{1} .
\end{gathered}
$$

We can write these equations in the form

$$
\mathbf{E}_{t}=\mathbf{W E}
$$

with the Cartan matrix

$$
\mathbf{W}=\left(\begin{array}{ccc}
0 & \pm \omega_{1} & \mp \omega_{2} \\
\omega_{1} & 0 & \omega_{0} \\
\omega_{2} & \omega_{0} & 0
\end{array}\right) \in \mathfrak{s o}(2,1)
$$

which belongs to the Lie algebra of the $S O(2,1)$ group of rotation and boost isometries in $\mathbb{R}^{2,1}$.

The flow equations (4.35) and the Frenet equations (4.24) of the parallel frame are related by the compatibility condition $\partial_{t}\left(\mathbf{E}_{x}\right)=\partial_{x}\left(\mathbf{E}_{t}\right)$, which is equivalent to a zero curvature equation (3.43) relating the Cartan matrices $\mathbf{W}$ and $\mathbf{U}$. Substituting these matrices (4.25) and (4.36) into equation (3.43), we obtain

$$
\begin{gathered}
u_{1 t}=\omega_{1 x}+u_{2} \omega_{0}, \quad u_{2 t}=\omega_{2 x}+u_{1} \omega_{0}, \\
\omega_{0 x}=\mp\left(u_{1} \omega_{2}-\omega_{1} u_{2}\right) .
\end{gathered}
$$

There is a similar compatibility condition $\partial_{x}\left(\vec{\gamma}_{t}\right)=\partial_{t}\left(\vec{\gamma}_{x}\right)$ relating the flow vector (4.32) and the tangent vector (4.3) of the curve, which is given by equation (3.47) using the notation (3.46). After substituting the matrices (4.25) and (4.36) along with the vectors (3.46) into this equation, we find that its tangential and normal components yield

$$
\begin{gathered}
h_{\| x}=-h_{1} u_{1}-h_{2} u_{2}, \\
\omega_{1}= \pm h_{1 x}+u_{1} h_{\|}, \quad \omega_{2}=\mp h_{2 x}+u_{2} h_{\|} .
\end{gathered}
$$

These compatibility equations (4.37), (4.38), (4.39), (4.40) are the Cartan structure equations of the parallel frame, describing all inelastic spacelike curve flows $\vec{\gamma}(t, x)$ with a non-null principal normal in $\mathbb{R}^{2,1}$.

\subsection{2. $S O(1,1)$-invariant formalism}

The gauge group for parallel frames consists of rigid $S O(1,1)$ hyperbolic rotations (4.31) acting on the normal vectors. Under the action of this group, the components of the Cartan matrix (4.25) along the curve are transformed by

$$
\begin{aligned}
& u_{1} \rightarrow u_{1} \cosh \phi-u_{2} \sinh \phi= \pm \kappa \cosh (\theta+\phi), \\
& u_{2} \rightarrow-u_{1} \sinh \phi+u_{2} \cosh \phi=\mp \kappa \sinh (\theta+\phi) .
\end{aligned}
$$


Similarly, the components of the Cartan matrix (4.36) along the flow are transformed by

$$
\begin{gathered}
\omega_{0} \rightarrow \omega_{0}, \\
\omega_{1} \rightarrow \omega_{1} \cosh \phi-\omega_{2} \sinh \phi, \\
\omega_{2} \rightarrow-\omega_{1} \sinh \phi+\omega_{2} \cosh \phi .
\end{gathered}
$$

Since the flow vector (4.32) is gauge invariant, its normal and tangential components in a parallel frame are transformed by

$$
\begin{gathered}
h_{\|} \rightarrow h_{\|}, \\
h_{1} \rightarrow h_{1} \cosh \phi+h_{2} \sinh \phi, \\
h_{2} \rightarrow h_{1} \sinh \phi+h_{2} \cosh \phi .
\end{gathered}
$$

A comparison with the case of rigid $S O(2)$ rotations in Sec. 3.3 suggests that we introduce a hyperbolic analog of complex variables.

Let $j$ denote a unit hyperbolic number (also called a split-complex number) defined by

$$
j^{2}=+1, \quad \bar{j}=-j
$$

in analogy to the complex imaginary number $i^{2}=-1, \bar{i}=-i$. The hyperbolic version of the Euler relation is

$$
\exp (j \theta)=\cosh \theta+j \sinh \theta
$$

If we write

$$
\begin{gathered}
u=u_{1}+j u_{2}= \pm \kappa \exp (-j \theta)= \pm \kappa \exp \left(-j \int \tau d x\right), \quad \omega=\omega_{1}+j \omega_{2}, \\
h_{\perp}= \pm\left(h_{1}-j h_{2}\right),
\end{gathered}
$$

then the rigid $S O(1,1)$ gauge transformations (4.41)-(4.45) become a hyperbolic phase rotation

$$
u \rightarrow u \exp (-j \phi), \quad \omega \rightarrow \omega \exp (-j \phi), \quad h_{\perp} \rightarrow h_{\perp} \exp (-j \phi) .
$$

We can now write the evolution equations in a $S O(1,1)$-invariant form:

$$
\begin{gathered}
u_{t}=\omega_{x}+j u \omega_{0}, \\
\omega_{0 x}=\mp \operatorname{Im}(\bar{u} \omega), \\
h_{\| x}=\mp \operatorname{Re}\left(\bar{u} h_{\perp}\right), \\
\omega=h_{\perp x}+u h_{\|} .
\end{gathered}
$$

Since $u$ is determined by the curve only up to rigid hyperbolic phase rotations (4.41), this splitcomplex variable $u$ is a Hasimoto variable which geometrically represents a $S O(1,1)$-covariant of the curve, in contrast to the invariants $\kappa, \tau$ which are determined uniquely by the curve.

This system (4.51)-(4.54) has an operator formulation, encoding a triple of $S O(1,1)$-invariant Hamiltonian operators, as follows. 
Theorem 8. For inelastic flows of spacelike curves with a non-null principal normal in $\mathbb{R}^{2,1}$, the curve covariant $u$ satisfies the $S O(1,1)$-invariant system

$$
\begin{aligned}
& u_{t}=D_{x} \omega \mp j u D_{x}^{-1} \operatorname{Im}(\omega \bar{u})=\mathscr{H}(\omega) \\
& \omega=D_{x} h_{\perp} \mp u D_{x}^{-1} \operatorname{Re}\left(\bar{u} h_{\perp}\right)=\mathscr{J}\left(h_{\perp}\right)
\end{aligned}
$$

where

$$
\begin{aligned}
\mathscr{H} & =D_{x} \pm j u D_{x}^{-1} \operatorname{Im}(u \mathscr{C}) \\
\mathscr{J} & =D_{x} \mp u D_{x}^{-1} \operatorname{Re}(u \mathscr{C})
\end{aligned}
$$

are a pair of compatible, $S O(1,1)$-invariant Hamiltonian and symplectic operators, and $\mathscr{C}$ denotes the complex conjugation operator. Moreover, the operators $\mathscr{H}$ and $\mathscr{J}$ are related by

$$
\mathscr{H} \mathscr{I}^{-1}=\mathscr{I} \mathscr{J}, \quad \mathscr{J} \mathscr{I}=\mathscr{I}^{-1} \mathscr{H}, \quad \mathscr{I}=j
$$

where $\mathscr{I}$ is is a Hamiltonian operator compatible with $\mathscr{H}$ and $\mathscr{J}$. Composition of these operators yields the $S O(1,1)$-invariant evolution equation

$$
u_{t}=\mathscr{R}^{2}\left(h_{\perp}\right)
$$

for $u$ in terms of the normal component $h_{\perp}$ of the flow, where

$$
\mathscr{R}=\mathscr{H} \mathscr{I}^{-1}=\mathscr{I} \mathscr{J}=j\left(D_{x} \mp u D_{x}^{-1} \operatorname{Re}(u \mathscr{C})\right)
$$

is a hereditary recursion operator.

This theorem is analogous to the similar result for timelike inelastic curve flows in Sec. 3.3 and has a similar proof by replacing $i$ with $j$.

An operator $\mathscr{D}$ here is Hamiltonian iff it defines an associated Poisson bracket (3.68) having the same structure as in the case of timelike inelastic curve flows, with $j$ in place of $i$. Similarly, based on Magri's general theorem [17], the $S O(1,1)$ invariance of the pair of compatible Hamiltonian operators $\mathscr{H}$ and $\mathscr{I}$ can be used here to derive a hierarchy of flows starting from a root flow $u_{t}=j u$ corresponding to the generator of $S O(1,1)$ hyperbolic phase-rotations on $u$. This leads to the following main result.

Theorem 9. There is a hierarchy of integrable tri-Hamiltonian flows on $u(t, x)$ given by

$$
\begin{aligned}
u_{t}=\mathscr{R}^{n+1}(j u) & =\mathscr{I}\left(\delta \mathfrak{H}^{(n+1)} / \delta \bar{u}\right)=\mathscr{H}\left(\delta \mathfrak{H}^{(n)} / \delta \bar{u}\right), \quad n=0,1,2, \ldots \\
& =\mathscr{E}\left(\delta \mathfrak{H}^{(n-1)} / \delta \bar{u}\right), \quad n=1,2, \ldots
\end{aligned}
$$

(called the $+n$ flow) in terms of Hamiltonians $\mathfrak{H}^{(n)}=\int H^{(n)} d x$ where

$$
\begin{aligned}
& \mathscr{I}=j, \quad \mathscr{H}=D_{x} \pm j u D_{x}^{-1} \operatorname{Im}(u \mathscr{C}), \\
& \mathscr{E}=\mathscr{R} \mathscr{H}=j\left(D_{x}^{2} \mp|u|^{2} \pm u D_{x}^{-1} \operatorname{Re}\left(u_{x} \mathscr{C}\right) \pm j u_{x} D_{x}^{-1} \operatorname{Im}(u \mathscr{C})\right)
\end{aligned}
$$

are compatible Hamiltonian operators, and where

$$
H^{(n)}=2(1+n)^{-1} D_{x}^{-1} \operatorname{Im}\left(\bar{u}(j \mathscr{H})^{n+1} u\right), \quad n=0,1,2, \ldots
$$

are local Hamiltonian densities. 
The expression for the Hamiltonians (4.64) in this theorem arises from a scaling formula derived in Ref. [1].

The +1 flow in the hierarchy (4.62) is explicitly given by

$$
u_{t}=j\left(u_{x x} \mp \frac{1}{2}|u|^{2} u\right)
$$

which is a variant of the defocusing NLS equation with $j$ in place of $i$. This equation (4.65) has the explicit tri-Hamiltonian structure

$$
u_{t}=\mathscr{I}\left(\delta \mathfrak{H}^{(2)} / \delta \bar{u}\right)=\mathscr{H}\left(\delta \mathfrak{H}^{(1)} / \delta \bar{u}\right)=\mathscr{E}\left(\delta \mathfrak{H}^{(0)} / \delta \bar{u}\right)
$$

in terms of the Hamiltonian densities

$$
H^{(0)}=|u|^{2}, \quad H^{(1)}=\operatorname{Im}\left(u_{x} \bar{u}\right), \quad H^{(2)}=-\left|u_{x}\right|^{2} \mp \frac{3}{4}|u|^{4}
$$

(modulo trivial densities given by total $x$-derivatives). Similarly, the +2 flow in the hierarchy (4.62) is given by

$$
u_{t}=u_{x x x} \mp \frac{3}{2}|u|^{2} u_{x}
$$

which is a variant of the complex defocusing $\mathrm{mKdV}$ equation, where $j$ replaces $i$. It has the triHamiltonian structure

$$
u_{t}=\mathscr{I}\left(\delta \mathfrak{H}^{(3)} / \delta \bar{u}\right)=\mathscr{H}\left(\delta \mathfrak{H}^{(2)} / \delta \bar{u}\right)=\mathscr{E}\left(\delta \mathfrak{H}^{(1)} / \delta \bar{u}\right)
$$

where $\mathfrak{H}^{(1)}$ and $\mathfrak{H}^{(2)}$ are given by the Hamiltonian densities (4.67), while $\mathfrak{H}^{(3)}$ is given by the Hamiltonian density

$$
H^{(3)}=\operatorname{Im}\left(u_{x} \bar{u}_{x x}\right) \pm \frac{3}{4}|u|^{2} \operatorname{Im}\left(u \bar{u}_{x}\right)
$$

(modulo trivial densities given by total $x$-derivatives).

In exactly the same way as for timelike inelastic curve flows, here the entire hierarchy (4.62) of flows corresponds to a hierarchy of commuting vector fields

$$
\mathrm{X}^{(n)}=\mathscr{R}^{n}(j u) \partial_{u}, \quad n=0,1,2, \ldots
$$

with the root vector field being the generator of hyperbolic phase-rotations $X^{(0)}=j u \partial_{u}$. All of the vector fields in the hierarchy are bi-Hamiltonian, with $\operatorname{pr} X^{(n)} \mathfrak{G}=\left\{\mathfrak{G}, \mathfrak{H}^{(n)}\right\}_{\mathscr{H}}=\left\{\mathfrak{G}, \mathfrak{H}^{(n+1)}\right\}_{\mathscr{I}}$ for $n \geq 0$, where $\mathfrak{H}^{(n)}=\int H^{(n)} d x$ is the functional with the Hamiltonian density (4.64), while each vector field for $n \geq 1$ is also tri-Hamiltonian, $\operatorname{pr}^{(n)} \mathfrak{G}=\left\{\mathfrak{G}, \mathfrak{H}^{(n-1)}\right\}_{\mathscr{E}}$. Since the entire hierarchy is commuting, every Hamiltonian vector field (4.71) is the generator of a symmetry for the defocusing NLS equation (4.65) as well as the defocusing mKdV equation (4.68), and every associated Hamiltonian (4.64) is a conserved density for both of these equations. 
Proposition 4. Each tri-Hamiltonian flow in the hierarchy (4.62) can be written in the form (4.60) as given by

$$
h_{\perp}=\mathscr{R}^{n-1}(j u), \quad n=1,2, \ldots
$$

Consequently, the $+n$ flow for $n \geq 1$ determines an inelastic timelike curve flow

$$
\vec{\gamma}_{t}=h_{\|}^{(n)} e_{\|}+h_{1}^{(n)} e_{1}+h_{2}^{(n)} e_{2} \quad n=1,2, \ldots
$$

whose tangential and normal components are given by

$$
h_{\|}^{(n)}=\frac{1}{2}(n-1) H^{(n-2)}, \quad h_{1}^{(n)}-j h_{2}^{(n)}= \pm \mathscr{R}^{n-1}(j u), \quad n=1,2, \ldots
$$

These equations of motions (4.73) can be written in an explicit form by use of the following variant of a complex parallel-frame notation:

$$
e_{\|}=T, \quad e_{\perp}=e_{1}+j e_{2}=e^{-j \theta}(N+j B)
$$

satisfying

$$
e_{\|} \times e_{\perp}=j e_{\perp}, \quad e_{\perp} \times \bar{e}_{\perp}=\mp j 2 e_{\|}
$$

and

$$
\eta\left(e_{\|}, e_{\|}\right)=1, \quad \eta\left(e_{\perp}, \bar{e}_{\perp}\right)=\mp 2, \quad \eta\left(e_{\|}, e_{\perp}\right)=\eta\left(e_{\perp}, e_{\perp}\right)=0 .
$$

The action of the gauge group (4.31) on these frame vectors consists of rigid hyperbolic phase rotations

$$
e_{\|} \rightarrow e_{\|}, \quad e_{\perp} \rightarrow e^{-j \phi} e_{\perp}
$$

where $\phi$ is constant.

The Frenet equations (4.24) of the underlying parallel frame become

$$
e_{\| x}= \pm \operatorname{Re}\left(\bar{u} e_{\perp}\right), \quad e_{\perp x}=u e_{\|},
$$

while the flow equations (4.35) are similarly given by

$$
e_{\| t}= \pm \operatorname{Re}\left(\bar{\omega} e_{\perp}\right), \quad e_{\perp t}=\omega e_{\|}+j \omega_{0} e_{\perp} .
$$

The equation of motions (4.73) for the curve then take the form

$$
\vec{\gamma}_{t}=h_{\|}^{(n)} e_{\|} \pm \operatorname{Re}\left(\bar{h}_{\perp}^{(n)} e_{\perp}\right), \quad n=1,2, \ldots
$$

where the tangential component $h_{\|}^{(n)}$ and the normal component $h_{\perp}^{(n)}= \pm\left(h_{1}^{(n)}-j h_{2}^{(n)}\right)$ are functions of the curve covariant $u$, the conjugate covariant $\bar{u}$, and their $x$-derivatives, as given by the expressions (4.74). Since $u \rightarrow e^{-j \phi} u$ and $\bar{u} \rightarrow e^{j \phi} \bar{u}$ under the gauge group (4.78), the tangential component is gauge invariant while the normal component is gauge equivariant,

$$
h_{\|}^{(n)} \rightarrow h_{\|}^{(n)}, \quad h_{\perp}^{(n)} \rightarrow e^{-j \phi} h_{\|}^{(n)} .
$$

Consequently, each equation of motion (4.32) is invariant under the isometry group $I S O(2,1)$ of $\mathbb{R}^{2,1}$ and thus describes a geometric non-stretching motion of the curve. 
The curve flow $n=1$ corresponding to the defocusing NLS equation (4.65) is determined by the components

$$
h_{\|}^{(1)}=0, \quad h_{\perp}^{(1)}=j u .
$$

Substituting these expressions into the $n=1$ equation of motion (4.81), we have $\vec{\gamma}_{t}=$ $\pm \operatorname{Re}\left(-j \bar{u} e_{\perp}\right)=\mp \operatorname{Re}\left(\bar{u} e_{\|} \times e_{\perp}\right)=-e_{\|} \times e_{\| x}$ by using the cross product identity (4.76) and the Frenet equations (4.79). Thus we obtain

$$
-\vec{\gamma}_{t}=T \times T_{x}=\vec{\gamma}_{x} \times \vec{\gamma}_{x x}, \quad \eta\left(\vec{\gamma}_{x}, \vec{\gamma}_{x}\right)=-1, \quad \eta\left(\vec{\gamma}_{x x}, \vec{\gamma}_{x x}\right) \neq 0
$$

which is a spacelike version of the vortex filament equation in Minkowski space $\mathbb{R}^{2,1}$.

The curve flow $n=2$ corresponding to the defocusing $\mathrm{mKdV}$ equation (4.68) has the components

$$
h_{\|}^{(2)}=\mp \frac{1}{2}|u|^{2}, \quad h_{\perp}^{(2)}=u_{x} .
$$

Substituting these expressions into the $n=2$ equation of motion (4.32), we find $\vec{\gamma}_{t}= \pm \operatorname{Re}\left(\bar{u}_{x} e_{\perp}\right) \mp$ $\frac{1}{2}|u|^{2} e_{\|}$. We simplify the term $\operatorname{Re}\left(\bar{u}_{x} e_{\perp}\right)=\operatorname{Re}\left(\bar{u} e_{\perp}\right)_{x}-\operatorname{Re}\left(\bar{u} e_{\perp x}\right)= \pm e_{\| x x}-|u|^{2} e_{\|}$by using the Frenet equations (4.79). Next we note $\eta\left(e_{\| x}, e_{\| x}\right)=\eta\left( \pm \operatorname{Re}\left(\bar{u} e_{\perp}\right), \pm \operatorname{Re}\left(\bar{u} e_{\perp}\right)\right)=\frac{1}{2} \eta\left(\bar{u} e_{\perp}, u \bar{e}_{\perp}\right)=$ $\mp|u|^{2}$ which follows from the relations (4.77). This yields $\vec{\gamma}_{t}=e_{\| x x} \mp \frac{3}{2}|u|^{2} e_{\|}$, and thus we obtain

$$
\vec{\gamma}_{t}=T_{x x} \mp \frac{3}{2} \eta\left(T_{x}, T_{x}\right) T=\vec{\gamma}_{x x x} \mp \frac{3}{2} \eta\left(\vec{\gamma}_{x x}, \vec{\gamma}_{x x}\right) \vec{\gamma}_{x}, \quad \eta\left(\vec{\gamma}_{x}, \vec{\gamma}_{x}\right)=1, \quad \eta\left(\vec{\gamma}_{x x}, \vec{\gamma}_{x x}\right) \neq 0
$$

which is a spacelike version of the non-stretching $\mathrm{mKdV}$ map equation [2] in Minkowski space $\mathbb{R}^{2,1}$.

\subsection{Spacelike curve flows with a null normal vector}

We now consider spacelike curves whose principal normal vector $T_{x}$ is assumed to be null

$$
\eta\left(T_{x}, T_{x}\right)=0
$$

at every point on the curve.

In contrast to the case when the principal normal is non-null, here the vectors $T_{x}$ and $T \times T_{x}$ are parallel, by the following argument. We note $\varepsilon\left(T \times T_{x}, T, T_{x}\right)=\eta\left(T \times T_{x}, T \times T_{x}\right)=$ $-\eta(T, T) \eta\left(T_{x}, T_{x}\right)=0$ from the properties (3.2), (3.3), and (3.5). Antisymmetry of $\varepsilon$ thereby implies $T \times T_{x}=a T+b T_{x}$ for some functions $a(x), b(x)$. Then, since $T \times T_{x}$ is orthogonal to $T$ from property (3.5), we find $0=\eta\left(T, T \times T_{x}\right)=a$ due to $\eta\left(T, T_{x}\right)=0$, and hence we have $T \times T_{x}=b T_{x}$.

To define a frame, we therefore need another vector, linearly independent of $T$ and $T_{x}$. Similarly to the case of null curves in 2 dimensions, it is natural to use a null vector on the opposite side of the lightcone in the normal plane $\mathbb{R}^{1,1}$ (which is orthogonal to $T$ ). If $\vec{v}$ is a null vector in the plane $\mathbb{R}^{1,1}$, let $\mathscr{N}$ be a linear map that produces a null vector $\mathscr{N}(\vec{v})$ such that $\eta(\mathscr{N}(\vec{v}), \vec{v})=-1$. Recall, from Sec. 2.2, that the null vectors $\vec{v}$ and $\mathscr{N}(\vec{v})$ are spatial reflections of each other with respect to the timelike vector $\vec{v}+\mathscr{N}(\vec{v})$ in $\mathbb{R}^{1,1}$, and that any change in the normalization $\eta(\mathscr{N}(\vec{v}), \vec{v})=-1$ only produces a scaling of the null vector $\mathscr{N}(\vec{v})$. 
A frame for a spacelike curve $\vec{\gamma}(x)$ with a null principal normal in $\mathbb{R}^{2,1}$ can then be defined by

$$
\begin{aligned}
& e_{\|}=\vec{\gamma}_{x}=T, \quad \text { spacelike tangent vector } \\
& e_{+}=\vec{\gamma}_{x x}=T_{x}, \quad \text { null normal vector } \\
& e_{-}=\mathscr{N}\left(\vec{\gamma}_{x x}\right)=\mathscr{N}\left(T_{x}\right), \quad \text { null opposite vector }
\end{aligned}
$$

with

$$
\begin{aligned}
& \eta\left(e_{ \pm}, e_{\|}\right)=0 \\
& \eta\left(e_{ \pm}, e_{ \pm}\right)=0 \\
& \eta\left(e_{+}, e_{-}\right)=-1
\end{aligned}
$$

where the properties (4.89)-(4.91) uniquely determine $e_{-}$when $e_{+}$and $e_{\|}$are given. Let

$$
\varepsilon=-\frac{1}{2} \varepsilon\left(e_{+}, e_{-}, e_{\|}\right)=\varepsilon\left(e_{0}, e_{1}, e_{\|}\right), \quad|\varepsilon|=1
$$

denote the orientation (equal to +1 or -1 ) of the frame vectors as given in terms of the volume form, where $e_{0}=\frac{1}{\sqrt{2}}\left(e_{+}+e_{-}\right)$is a unit timelike vector and $e_{1}=\frac{1}{\sqrt{2}}\left(e_{+}-e_{-}\right)$is a unit spacelike vector determined by the null normal vectors in the frame.

The Frenet equations for this frame (4.88) are easily derived by the same steps used in the case of null curves in 2 dimensions. First, from (4.88b) we have

$$
e_{\| x}=e_{+} .
$$

Next, from the $x$-derivative of equations (4.89) and (4.90), we have $0=\eta\left(e_{+x}, e_{+}\right)$and $0=$ $\eta\left(e_{+x}, e_{\|}\right)$after using equation (4.93). Thus we get

$$
e_{+x}=\sigma e_{+}
$$

for some function $\sigma(x)$. Then, similarly we obtain $\eta\left(e_{-x}, e_{-}\right)=0, \eta\left(e_{-x}, e_{\|}\right)=1, \eta\left(e_{-x}, e_{+}\right)=\sigma$, by using equations (4.91), (4.93), and (4.94). This yields

$$
e_{-x}=e_{\|}-\sigma e_{-} .
$$

Therefore, the Frenet equations are given by

$$
\left(\begin{array}{l}
e_{\|} \\
e_{+} \\
e_{-}
\end{array}\right)_{x}=\left(\begin{array}{ccc}
0 & 1 & 0 \\
0 & \sigma & 0 \\
1 & 0 & -\sigma
\end{array}\right)\left(\begin{array}{l}
e_{\|} \\
e_{+} \\
e_{-}
\end{array}\right)
$$

where the Cartan matrix $\left(\begin{array}{ccc}0 & 1 & 0 \\ 0 & \sigma & 0 \\ 1 & 0 & -\sigma\end{array}\right) \in \mathfrak{s o}(2,1)$ of the frame (4.88) belongs to the Lie algebra of the $S O(2,1)$ group of rotation and boost isometries in $\mathbb{R}^{2,1}$. These equations (4.96) are preserved if the normalization (4.91) of the null frame is changed. Therefore, $\sigma$ geometrically represents a Lorentzian curvature invariant of the curve.

A general frame for a spacelike curve in $\mathbb{R}^{2,1}$ is related to this Frenet frame by the action of arbitrary $x$-dependent $S O(2,1)$ rotations and boosts applied to the vectors $(4.88)$. If the tangent vector $T$ is preserved as one of the frame vectors, then the resulting frame is given by applying a 
general $x$-dependent $S O(1,1)$ boost on the null normal vectors $e_{ \pm}$in the $\mathbb{R}^{1,1}$ normal plane of the curve. This yields the boosted frame

$$
\mathbf{E}=\left(\begin{array}{c}
e_{\|} \\
\tilde{e}_{+} \\
\tilde{e}_{-}
\end{array}\right)=\left(\begin{array}{c}
e_{\|} \\
\exp (\theta) e_{+} \\
\exp (-\theta) e_{-}
\end{array}\right)
$$

where the boost acts as a scaling, parameterize by $\theta(x)$, and where the frame vectors satisfy the orthogonality relations

$$
\begin{gathered}
\eta\left(e_{\|}, \tilde{e}_{ \pm}\right)=\eta\left(\tilde{e}_{ \pm}, \tilde{e}_{ \pm}\right)=0 \\
\eta\left(e_{\|}, e_{\|}\right)=-\eta\left(\tilde{e}_{+}, \tilde{e}_{-}\right)=1
\end{gathered}
$$

and the cross product relations

$$
\tilde{e}_{+} \times \tilde{e}_{-}=-2 \varepsilon e_{\|}, \quad e_{\|} \times \tilde{e}_{ \pm}= \pm 2 \varepsilon e_{ \pm} .
$$

To derive the Cartan matrix of the boosted frame (4.97), we first take the $x$-derivative of the frame vectors, and then we substitute the Frenet equations (4.96) followed by

$$
\left(\begin{array}{l}
e_{\|} \\
e_{+} \\
e_{-}
\end{array}\right)=\left(\begin{array}{c}
e_{\|} \\
\exp (-\theta) \tilde{e}_{+} \\
\exp (\theta) \tilde{e}_{-}
\end{array}\right)
$$

which yields

$$
\left(\begin{array}{c}
e_{\|} \\
\tilde{e}_{+} \\
\tilde{e}_{-}
\end{array}\right)_{x}=\left(\begin{array}{ccc}
0 & \exp (-\theta) & 0 \\
0 & \sigma+\theta_{x} & 0 \\
\exp (-\theta) & 0 & -\theta_{x}-\sigma
\end{array}\right)\left(\begin{array}{c}
e_{\|} \\
\tilde{e}_{+} \\
\tilde{e}_{-}
\end{array}\right) .
$$

We now see that, similarly to the case of a spacelike curve with a non-null principal normal, here the gauge freedom in equation (4.102) can be used to define a Lorentzian parallel frame by the geometrical condition that the $x$-derivative of each null normal vector $e_{ \pm}$is parallel to the tangent vector $e_{\|}$. This condition can be achieved if (and only if)

$$
\theta_{x}=-\sigma
$$

The resulting frame given by equations (4.97) and (4.103) is a parallel frame whose Cartan matrix is given by

$$
\mathbf{E}_{x}=\mathbf{U E}
$$

where

$$
\mathbf{U}=\left(\begin{array}{lll}
0 & u & 0 \\
0 & 0 & 0 \\
u & 0 & 0
\end{array}\right) \in \mathfrak{s o}(2,1)
$$

with

$$
u=\exp (-\theta)=\exp \left(-\int \sigma d x\right)
$$


Note that the Cartan matrix (4.105) has one fewer component than in the case of a spacelike curve with a non-null principal normal, since here the principal normal is constrained to lie on the lightcone. In particular, this Cartan matrix belongs to a 1-dimensional subalgebra consisting of a boost combined with a rotation, which lies in a subspace of the perp space $\mathfrak{s o}(1,1)_{\perp} \simeq \mathbb{R}^{1,1}$ of the stabilizer subalgebra $\mathfrak{s o}(1,1) \subset \mathfrak{s o}(2,1)$ of the frame vector $e_{\|}$.

A parallel frame is unique up to $x$-independent (rigid) boosts given by

$$
\theta \rightarrow \theta+\phi
$$

where $\phi$ is constant. Under these transformations, the tangent vector $e_{\|}$is preserved, while the null normal vectors $\tilde{e}_{ \pm}$are rigidly scaled

$$
\tilde{e}_{+} \rightarrow \exp (\phi) \tilde{e}_{+}, \quad \tilde{e}_{-} \rightarrow \exp (-\phi) \tilde{e}_{-}
$$

Any two parallel frames related by this transformation are gauge equivalent, and thus the $S O(1,1)$ group of these rigid boosts thereby defines the gauge (equivalence) group for parallel frames. Stated another way, a parallel frame for a spacelike curve with a null principal normal is determined only up to the action of this gauge group.

\subsubsection{Inelastic Flow Equations}

Let $\vec{\gamma}(t, x)$ be a spacelike inelastic curve flow that locally preserves the proper distance normalization (3.9) of the arclength parameter and the null signature (4.87) of the principal normal. Such flows are specified by a flow vector

$$
\vec{\gamma}_{t}=h_{\|} e_{\|}+h_{+} \tilde{e}_{+}+h_{-} \tilde{e}_{-}
$$

expressed in terms of a tangential component $h_{\|}$and a pair of normal components $h_{ \pm}$with respect to the frame vectors $e_{\|}, \tilde{e}_{ \pm}$.

The parallel frame will be carried by the flow, such that the orthogonality relations (4.89)-(4.91) are preserved. This implies that the $t$-derivative of the frame vectors $e_{\|}, \tilde{e}_{ \pm}$is given by

$$
\begin{gathered}
e_{\| t}=\omega_{+} \tilde{e}_{+}+\omega_{-} \tilde{e}_{-}, \\
\tilde{e}_{+t}=\omega_{-} e_{\|}+\omega_{0} \tilde{e}_{+}, \quad \tilde{e}_{-t}=\omega_{+} e_{\|}-\omega_{0} \tilde{e}_{-} .
\end{gathered}
$$

We can write these equations in the form

$$
\mathbf{E}_{t}=\mathbf{W E}
$$

with the Cartan matrix

$$
\mathbf{W}=\left(\begin{array}{ccc}
0 & \omega_{+} & \omega_{-} \\
\omega_{-} & \omega_{0} & 0 \\
\omega_{+} & 0 & -\omega_{0}
\end{array}\right) \in \mathfrak{s o}(2,1)
$$

which belongs to the Lie algebra of the $S O(2,1)$ group of rotation and boost isometries in $\mathbb{R}^{2,1}$. 
Under the action of the gauge group of the parallel frame, the Cartan matrix (4.105) along the curve is scaled by

$$
u \rightarrow \exp (-\phi) u
$$

while the Cartan matrix (4.113) along the flow is similarly scaled by

$$
\begin{gathered}
\omega_{0} \rightarrow \omega_{0}, \\
\omega_{ \pm} \rightarrow \exp (\mp \phi) \omega_{ \pm} .
\end{gathered}
$$

Since the flow vector (4.109) is gauge invariant, its normal and tangential components also scale by

$$
\begin{gathered}
h_{\|} \rightarrow h_{\|}, \\
h_{ \pm} \rightarrow \exp (\mp \phi) h_{ \pm} .
\end{gathered}
$$

The flow equations (4.112) and the Frenet equations (4.104) of the parallel frame are related by the compatibility condition $\partial_{t}\left(\mathbf{E}_{x}\right)=\partial_{x}\left(\mathbf{E}_{t}\right)$, which is equivalent to a zero curvature equation (3.43) relating the Cartan matrices $\mathbf{W}$ and $\mathbf{U}$. Substituting these matrices (4.105) and (4.113) into equation (3.43), we obtain

$$
\begin{gathered}
\omega_{-x}=0, \\
\omega_{0 x}=-u \omega_{-}, \\
u_{t}=\omega_{+x}-u \omega_{0} .
\end{gathered}
$$

A similar compatibility condition $\partial_{x}\left(\vec{\gamma}_{t}\right)=\partial_{t}\left(\vec{\gamma}_{x}\right)$ relates the flow vector (4.109) and the tangent vector (4.3) of the curve, as formulated by equation (3.47) using the notation (3.46). After substituting the matrices (4.105) and (4.113) along with the vectors (3.46) into this equation, we find that its tangential and normal components yield

$$
\begin{gathered}
h_{\| x}=-u h_{-}, \\
\omega_{-}=h_{-x}, \\
\omega_{+}=h_{+x}+u h_{\|} .
\end{gathered}
$$

These compatibility equations (4.119)-(4.121) and (4.122)-(4.124) are the Cartan structure equations of the parallel frame, describing all inelastic spacelike curve flows $\vec{\gamma}(t, x)$ with a null principal normal in $\mathbb{R}^{2,1}$. Compared to all of the previous cases, here the system (4.119)-(4.124) has a quite different operator structure, as follows.

From equations (4.119) and (4.123), we have

$$
h_{-}=a x+b, \quad \omega_{-}=a
$$

for some functions $a(t), b(t)$. Next, equation (4.120) yields

$$
\omega_{0}=-a v, \quad v=\int u d x
$$

and then equation (4.122) similarly yields

$$
h_{\|}=-(a x+b) v+a w, \quad w=\int v d x .
$$


Substituting these expressions into equation (4.124), we get

$$
\omega_{+}=h_{+x}+\left(a w-(a x+b) w_{x}\right) w_{x x} .
$$

Finally, from equation (4.121), we obtain

$$
u_{t}=w_{t x x}=\left(h_{+}+a w w_{x}-\frac{1}{2}(a x+b) w_{x}^{2}\right)_{x x}
$$

which directly implies

$$
w_{t}=h_{+}+a w w_{x}-\frac{1}{2}(a x+b) w_{x}^{2}+c x+d
$$

for some functions $c(t), d(t)$. Hence we have derived the following result.

Theorem 10. For inelastic flows of spacelike curves with a null principal normal in $\mathbb{R}^{2,1}$, the curve invariant $u$ satisfies the system

$$
w_{t}=h_{+}+a w w_{x}-\frac{1}{2}(a x+b) w_{x}^{2}+c x+d, \quad w_{x}=v, \quad v_{x}=u
$$

for $w$ in terms of the normal component $h_{+}$of the flow, where $a(t), b(t), c(t), d(t)$ are arbitrary functions.

We will now restrict attention to the class of flows that exhibit invariance under $x$-translations. This implies $a=c=0$, so thus the system (4.131) reduces to

$$
\begin{aligned}
& w_{t}+\frac{1}{2} w_{x}^{2}=h_{+}, \\
& w_{x x}=u,
\end{aligned}
$$

or equivalently

$$
\begin{aligned}
& v_{t}+v v_{x}=D_{x} h_{+}, \\
& v_{x}=u,
\end{aligned}
$$

after a transformation $t \rightarrow \tilde{t}=\int b d t$ and $h_{+} \rightarrow \tilde{h}_{+}=\left(h_{+}+d\right) / b$, when $b \neq 0$. The form of this system (4.134)-(4.135) is closely related to both Burgers' equation and the KdV equation. Consider, first, the flow generated by

$$
h_{+}=-v_{x}=-w_{x x} .
$$

This yields Burgers' equation

$$
v_{t}+v v_{x}+v_{x x}=0
$$

for $u=\int v d x$, or equivalently

$$
w_{t}+\frac{1}{2} w_{x}^{2}+w_{x x}=0
$$

in potential form, for $v=\int w d x$. We recall that, from Sec. 2.2, Burgers' equation has a gradientenergy structure,

$$
v_{t}=-D_{x}\left(\exp (w) D_{x} \delta \mathfrak{H} / \delta v\right)
$$


and in potential form

$$
w_{t}=\exp (w) \delta \mathfrak{H} / \delta w
$$

with $\mathfrak{H}=\int H d x$ where

$$
H=\frac{1}{2} \exp (-w) v^{2}
$$

is the energy density. Next, consider the flow generated by

$$
h_{+}=-v_{x x}=-w_{x x x} .
$$

This yields the $\mathrm{KdV}$ equation

$$
v_{t}+v v_{x}+v_{x x x}=0
$$

for $u=\int v d x$, or equivalently in potential form,

$$
w_{t}+\frac{1}{2} w_{x}^{2}+w_{x x x}=0
$$

for $v=\int w d x$. The KdV equation has the Hamiltonian structure,

$$
v_{t}=\mathscr{H}(\mathfrak{H} / \delta v)
$$

with $\mathfrak{H}=\int H d x$ where

$$
H=\frac{1}{2} v_{x}^{2}-\frac{1}{6} v^{3}
$$

is the Hamiltonian density, and where

$$
\mathscr{H}=D_{x}
$$

is a Hamiltonian operator. Equivalently, this structure has the potential form

$$
w_{t}=-\mathscr{D}(\boldsymbol{\delta} \mathfrak{H} / \delta w)
$$

where

$$
\mathscr{D}=D_{x}^{-1}
$$

is a Hamiltonian operator.

Both Burgers' equation (4.137) and the KdV equation (4.143) are integrable systems. However, their respective recursion operators do not appear in the underlying $x$-translation invariant system (4.134)-(4.135), or (4.132)-(4.133), describing the class of flows (4.131) with $a=0$ and $b \neq 0$.

Finally, we consider the class of flows with $a=b=c=d=0$,

$$
w_{t}=h_{+}, \quad w_{x}=v,
$$

or equivalently

$$
v_{t}=D_{x} h_{+}, \quad v_{x}=u .
$$

These flows give rise to a hierarchy of linear flows generated by a recursion operator $\mathscr{R}=D_{x}$, starting from the root flow $h_{+}=v_{x}=w_{x x}$. Hence we have the following result. 
Theorem 11. There is a hierarchy of integrable linear flows on $v(t, x)$ given by

$$
\begin{aligned}
v_{t}=h_{+}^{(n)} & =\mathscr{R}^{n}\left(v_{x}\right), \quad n=1,2, \ldots \\
& = \begin{cases}-\delta \mathfrak{H}^{(l)} / \delta v, \quad l=(n+1) / 2, & n=1,3, \ldots \\
-D_{x}\left(\delta \mathfrak{H}^{(l)} / \delta v\right), \quad l=n / 2, & n=2,4, \ldots\end{cases}
\end{aligned}
$$

in terms of energies/Hamiltonians $\mathfrak{H}^{(l)}=\int H^{(l)} d x$, where

$$
H^{(l)}=\frac{1}{2}(-1)^{l-1}\left(D_{x}^{l} v\right)^{2} \quad l=1,2, \ldots
$$

are local densities, and where

$$
\mathscr{R}=D_{x}
$$

is a recursion operator.

This theorem is a direct counterpart of the hierarchy of linear flows (2.68) shown in Theorem 5 for the case of null curves in 2 dimensions.

The +1 flow in the hierarchy (4.152) is given by the heat equation

$$
v_{t}=v_{x x}
$$

which has the gradient structure

$$
v_{t}=-\delta \mathfrak{H}^{(1)} / \delta v
$$

in terms of the energy density

$$
H^{(1)}=\frac{1}{2} v_{x}^{2} .
$$

All of the odd flows in this hierarchy have a similar structure.

The +2 flow in the hierarchy (4.152) is given by

$$
v_{t}=v_{x x x}
$$

which is the Airy equation. This equation has the Hamiltonian structure

$$
v_{t}=-\mathscr{H}\left(\delta \mathfrak{H}^{(1)} / \delta v\right)
$$

where the Hamiltonian $\mathfrak{H}^{(1)}$ is the same expression as the energy integral appearing in the +1 flow, and where $\mathscr{H}$ is the Hamiltonian operator (4.147). There is a similar structure for all of the even flows in the hierarchy.

To conclude, we now work out the underlying equations of motion for the curve flows determined by the preceding flows. We begin by using the Frenet relations (4.88) to express the frame vectors (4.97) in terms of the tangent vector $\vec{\gamma}_{x}$ :

$$
\begin{aligned}
& e_{\|}=\vec{\gamma}_{x} \\
& \tilde{e}_{+}=u^{-1} e_{\| x}=u^{-1} \vec{\gamma}_{x x} \\
& \tilde{e}_{-}=u \mathscr{N}\left(e_{\| x}\right)=u \mathscr{N}\left(\vec{\gamma}_{x x}\right)
\end{aligned}
$$

where, recall, $\mathscr{N}$ is the linear map on null vectors in the Minkowski plane orthogonal to $e_{\|}$in $\mathbb{R}^{2,1}$. In particular, for any null vector $v$ such that $\eta\left(e_{\|}, v\right)=0$, this map is uniquely determined by the 
properties

$$
\eta\left(\mathscr{N}(\vec{v}), e_{\|}\right)=0, \quad \eta(\mathscr{N}(\vec{v}), \vec{v})=-1, \quad \eta(\mathscr{N}(\vec{v}), \mathscr{N}(\vec{v}))=0 .
$$

We next derive an expression for the curve invariant $u$ in terms of $x$-derivatives of these frame vectors. From the Frenet equations (4.104) of the frame, we have $e_{\| x x}=u_{x} \tilde{e}_{+}=u_{x} u^{-1} e_{\| x}$. Then, since $e_{\| x}$ is a null vector orthogonal to $e_{\|}$, we obtain

$$
(\ln u)_{x}=-\eta\left(e_{\| x x}, \mathscr{N}\left(e_{\| x}\right)\right)=-\eta\left(\vec{\gamma}_{x x x}, \mathscr{N}\left(\vec{\gamma}_{x x}\right)\right)
$$

and hence

$$
u=\int \exp \left(-\eta\left(\vec{\gamma}_{x x x}, \mathscr{N}\left(\vec{\gamma}_{x x}\right)\right)\right) d x .
$$

The flow vector (4.109) now leads to the following results.

Proposition 5. The flows (4.136) and (4.142) producing, respectively, Burgers' equation (4.137) and the KdV equation (4.143) determine an inelastic spacelike curve flow with a null principal normal, where the components of the flow vector (4.109) are given by

$$
\begin{gathered}
h_{\|}=-v=-\int u d x, \quad h_{-}=1 \\
h_{+}=-u \quad \text { and } \quad h_{+}=-u_{x}
\end{gathered}
$$

where $u$ is the curve covariant (4.166) and $v$ is its potential.

The resulting curve flows are explicitly given by

$$
\vec{\gamma}_{t}=-v \vec{\gamma}_{x}+u \mathscr{N}\left(\vec{\gamma}_{x x}\right)-\vec{\gamma}_{x x}, \quad \eta\left(\vec{\gamma}_{x}, \vec{\gamma}_{x}\right)=1, \quad \eta\left(\vec{\gamma}_{x x}, \vec{\gamma}_{x x}\right)=0
$$

which corresponds to Burgers' equation (4.137), and

$$
\vec{\gamma}_{t}=-v \vec{\gamma}_{x}+u \mathscr{N}\left(\vec{\gamma}_{x x}\right)-\vec{\gamma}_{x x x}, \quad \eta\left(\vec{\gamma}_{x}, \vec{\gamma}_{x}\right)=1, \quad \eta\left(\vec{\gamma}_{x x}, \vec{\gamma}_{x x}\right)=0
$$

which corresponds to the KdV equation (4.143).

Proposition 6. In the hierarchy (4.152), each flow determines an inelastic spacelike curve flow with a null principal normal, where the components of the flow vector (4.109) are given by

$$
h_{\|}=0, \quad h_{-}=0, \quad h_{+}^{(n)}=\mathscr{R}^{n}\left(v_{x}\right)=D_{x}{ }^{n} u, \quad n=1,2, \ldots
$$

in terms of the curve covariant (4.166).

The curve flow $n=1$ corresponding to the heat equation (4.156) is explicitly given by

$$
\vec{\gamma}_{t}=\vec{\gamma}_{x x}, \quad \eta\left(\vec{\gamma}_{x}, \vec{\gamma}_{x}\right)=1, \quad \eta\left(\vec{\gamma}_{x x}, \vec{\gamma}_{x x}\right)=0
$$

which is a non-stretching variant of the heat map equation, in Minkowski space $\mathbb{R}^{2,1}$. Similarly, the curve flow $n=2$ corresponding to the Airy equation (4.159) is given by

$$
\vec{\gamma}_{t}=\vec{\gamma}_{x x x} \quad \eta\left(\vec{\gamma}_{x}, \vec{\gamma}_{x}\right)=1, \quad \eta\left(\vec{\gamma}_{x x}, \vec{\gamma}_{x x}\right) \neq 0
$$

which is a non-stretching Airy map equation in Minkowski space $\mathbb{R}^{2,1}$.

Note that all of these equations of motion (4.169), (4.170), (4.172), (4.173) are invariant under the isometry group $\operatorname{ISO}(2,1)$ of $\mathbb{R}^{2,1}$ and thus describe geometric non-stretching motions of the curve. 


\section{Conclusion}

There are several interesting directions in which the methods and results in this paper can be extended.

Firstly, one can consider inelastic curve flows in $n$-dimensional Minkowski space $\mathbb{R}^{n, 1}$ for $n \geq 4$. Inelastic flows of timelike curves will yield an integrable multi-component $S O(n-1)$-invariant vector version of the defocusing $\mathrm{mKdV}$ equation and its bi-Hamiltonian integrability structure, while spacelike curves will similarly yield a $S O(n-2,1)$-invariant variant of that equation. Inelastic flows of null curves will yield multi-component Burgers' equations and their symmetry-integrability structures.

Secondly, one can consider inelastic curve flows in Lorentzian symmetry spaces $M=G / S O(n-$ $1,1)$, with $G=S O(n, 1)$ and $G=S U(n-1,1)$ for $n \geq 3$. These two spaces are curved generalizations of Minkowski space in which their frame bundles have the same $S O(n-1,1)$ gauge group of rotations and boosts as in the flat space $\mathbb{R}^{n, 1}$. The space $M=S O(n, 1) / S O(n-1,1)$ will yield the same multi-component vector defocusing $\mathrm{mKdV}$ equations obtained from $\mathbb{R}^{n, 1}$, whereas the space $M=S U(n-1,1) / S O(n-1,1)$ will yield different multi-component vector $\mathrm{mKdV}$ equations, analogously to the geometric origin of the two known multi-component vector focusing $\mathrm{mKdV}$ equations from [2] the Riemannian symmetric spaces $M=G / S O(n)$, with $G=S O(n+1)$ and $G=S U(n)$ for $n \geq 3$. For $n=3$, the Lorentzian symmetric space $M=S O(3,1) / S O(2,1)$ will yield the same two integrable defocusing NLS equations as found in this paper, while the space $M=S U(2,1) / S O(2,1)$ will yield two other integrable defocusing NLS equations (and their bi-Hamiltonian integrability structures).

Lastly, one can also obtain integrable sinh-Gordon equations, including (split) complex versions and multi-component vector versions, by considering inelastic curve flows related to non-stretching wave map equations in $M=S O(n, 1) / S O(n-1,1)$ and $M=S U(n-1,1) / S O(n-1,1)$, in analogy with the various versions of sine-Gordon equations obtained from [2,3] the Riemannian symmetric spaces $M=S O(n+1) / S O(n)$ and $M=S U(n) / S O(n)$, for $n \geq 3$.

\section{Acknowledgments}

S.C.A. is supported by an NSERC grant.

\section{References}

[1] S.C. Anco, Conservation laws of scaling-invariant field equations, J. Phys. A: Math. and Gen. 36 (2003), $8623-8638$.

[2] S.C. Anco, Hamiltonian flows of curves in symmetric spaces $\mathrm{G} / \mathrm{SO}(\mathrm{N})$ and vector soliton equations of $\mathrm{mKdV}$ and sine-Gordon type, SIGMA 2 (2006), 044 (18 pages).

[3] S.C. Anco, Group-invariant soliton equations and bi-Hamiltonian geometric curve flows in Riemannian symmetric spaces, J. Geom. Phys. 58 (2008), 1-37.

[4] S.C. Anco and R. Myrzakulov, Integrable generalizations of Schrodinger maps and Heisenberg spin models from Hamiltonian flows of curves and surfaces, J. Geom. Phys. 60 (2010), 1576-1603.

[5] R. Bishop, There is more than one way to frame a curve, Amer. Math. Monthly 82 (1975), 246-251.

[6] P. Bracken and J. Hayes, On a formulation of certain integrable systems using hyperbolic numbers, Phys. Lett. A 301 (2002), 191-194.

[7] F. Catoni, D. Boccaletti, F. Cannata, V. Catoni V and P. Zampetti, Geometry of Minkowski Space-Time (Springer, 2011).

[8] Q. Ding and J. Inoguchi, Chaos Solitons Fractals 21 (2004), 669-677. 
[9] Q. Ding, W. Wang and Y. Wang, A motion of spacelike curves in the Minkowski 3-space and the KdV equation, Phys. Lett. A 374 (2010), 3201-3205.

[10] A. Fujioka and T. Kurose, Motions of curves in the complex hyperbola and the Burgers hierarchy, Osaka J. Math. 45 (2008), 1057-1065.

[11] Y. Fukumoto and T. Miyazaki, Three-dimensional distortions of a vortex filament with axial velocity, J. Fluid Mech. 222 (1991), 369-416.

[12] R. Goldstein and D. Petrich, The Korteweg-de Vries hierarchy as dynamics of closed curves in the plane, Phys. Rev. Lett. 67 (1991), 3203-3206.

[13] H. Guggenheimer, Differential Geometry (McGraw Hill, 1963).

[14] N. Gurbuz, Inextensible flows of spacelike, timelike and null curves, Int. J. Contemp. Math. Sciences 4 (2009), 1599-1604.

[15] H. Hasimoto, A soliton on a vortex filament, J. Fluid Mech. 51 (1972), 477-485.

[16] G.L. Lamb, Solitons on moving space curves, J. Math. Phys. 18 (1977), 1654-1661.

[17] F. Magri, A simple model of the integrable Hamiltonian equation, J. Math. Phys. 19 (1978), 1156-1162.

[18] G. Mari Beffa G, J. Sanders and J.-P. Wang, Integrable systems in three-dimensional Riemannian geometry, J. Nonlinear Sci. 12 (2002), 143-167.

[19] E. Musso and K. Nicolodi, Hamiltonian flows on null curves. Nonlinearity 23 (2010), 2117-2129.

[20] K. Nakayama, Motion of curves in hyperboloid in the Minkowski space, J. Phys. Soc. Jpn. 67 (1998), 3031-3037.

[21] B. O’Neill, Semi-Riemannian Geometry With Applications to Relativity (Elsevier, 1983).

[22] M. Ozdemir M and A.A. Ergin, Parallel frames on non-lightlike curves, Missouri J. Math. Sci. 20 (2008) 127-137.

[23] J. Sanders and J.-P. Wang, Integrable systems in n-dimensional Riemannian geometry, Moscow Math. J. 3 (2003), 1369-1393. 